\title{
Well-Posedness and Long Time Behavior of the Non-Isothermal Viscous Cahn-Hilliard Equation with Dynamic Boundary Conditions
}

\author{
Ciprian Gal
}

\author{
Communicated by Y. Charles Li, received December 2, 2007.
}

\begin{abstract}
We consider a model of non-isothermal phase transition taking place in a confined container. The order parameter $\phi$ is governed by a CahnHilliard type equation which is coupled with a nonlinear heat equation for the temperature $\theta$. The former is subject to a nonlinear dynamic boundary condition recently proposed by some physicists to account for interactions of the material with the walls. The latter is endowed with a boundary condition which can be a standard one (Dirichlet, Neumann or Robin). We thus formulate a class of initial and boundary value problems whose local existence and uniqueness is proven by means of a Faedo-Galerkin approximation scheme. The local solution becomes global owing to suitable a priori estimates. Then we analyze the asymptotic behavior of the solutions within the theory of infinite-dimensional dynamical systems. In particular, we demonstrate the existence of a finite dimensional global attractor as well as of an exponential attractor.
\end{abstract}

\section{CONTENTS}

1. Introduction 40

2. Preliminary results 43

3. Uniform a priori estimates and existence of solutions 46

4. Global and exponential attractors $\quad 60$

$\begin{array}{ll}\text { References } & 65\end{array}$

1991 Mathematics Subject Classification. 35K55, 74N20, 35B40, 35B45, 37L30.

Key words and phrases. Viscous Cahn-Hilliard equation, dynamic boundary conditions, global attractors, exponential attractors, non-isothermal Cahn-Hilliard equations. 


\section{Introduction}

The viscous Cahn-Hilliard equation

$$
\partial_{t} \phi+k_{1} \Delta\left(\rho \Delta \phi-F_{1}^{\prime}(\phi)-\alpha \partial_{t} \phi\right)=0, \alpha, \rho, k_{1}>0,
$$

is a very important equation in materials science that describes spinodal decomposition, in absence of mechanical stresses, of binary mixtures that appears, for example, in cooling processes of alloys, glasses or polymer mixtures (see [12], [37], $[\mathbf{4 3}],[\mathbf{4 4}]$ and the references cited therein). Here $\phi$ is the relative concentration difference of the mixture components, $\rho$ is related to the surface tension at the interface, $k_{1}$ is the mobility, $\alpha>0$ is a viscous parameter. We also mention that the viscosity term can be interpreted as describing the influences of internal microforces (see e.g., $[\mathbf{3 3}]$ ). Moreover, $F_{1}$ is a potential which accounts for the presence of different phases. For instance, $F_{1}$ can be a logarithmic potential which is usually approximated by a double well potential, i.e., $F_{1}(s)=\frac{1}{4}\left(s^{2}-1\right)^{2}$. For simplicity, we shall take $\rho=k_{1}=1$ in what follows.

The mathematical literature regarding (1.1) is rather vast. Now, the usual boundary conditions considered for problem (1.1) are Neumann and periodic boundary conditions. Equation (1.1) is supplemented by the initial condition $\phi(0, x)=$ $\phi_{0}(x)$ in $\Omega$, where $\Omega$ is a bounded domain in $\mathbf{R}^{3}$ with smooth boundary $\partial \Omega:=\Gamma$. For the Cauchy problem (1.1) with Neumann boundary conditions, the results on global existence, uniqueness and large time behavior of solutions have been established in $[\mathbf{1 7}],[\mathbf{1 8}],[\mathbf{3 8}],[\mathbf{5 1}],[\mathbf{5 4}],[\mathbf{5 5}]$. For the sake of simplicity, we refer the reader to [16], where plenty of references are properly quoted. However, in recent years, physicists have considered the study of phase separation in confined systems. In this case, one has to account for the dynamic interactions of the material with the walls (see, e.g., $[\mathbf{2 0}],[\mathbf{2 1}]$ and their references), which leads to additional terms in the free energy and then to dynamic boundary conditions, in the sense that the term $\partial_{t} \phi$ appears in the boundary conditions. As a consequence, one deduces a dynamic boundary condition of the form

$$
\partial_{t} \phi=d \Delta_{\Gamma} \phi-\partial_{\mathbf{n}} \phi-\beta \phi
$$

on $\Gamma \times(0,+\infty)$. Here the constants $d, \beta>0$ and $\Delta_{\Gamma}$ denotes the Laplace-Beltrami operator on the surface $\Gamma$. We recall that, phenomenologically speaking, the boundary condition (1.2) means that the density at the surface relaxes towards equilibrium with a rate proportional to the driving force given by the Frechét derivative of the free energy functional (see $[\mathbf{2 2}],[\mathbf{2 3}]$ ). Such problems (1.1)-(1.2), supplemented with a no-flux condition on the chemical potential $\mu=\alpha \partial_{t} \phi-\Delta \phi+F_{1}^{\prime}(\phi)$, have recently been studied in a series of interesting papers where satisfactory results on the global existence and uniqueness of solutions, as well as results on the long time behavior of solutions have been obtained. For instance, R. Racke \& S. Zheng [48] show the existence and uniqueness of a global solution to this problem, and later J. Prüss, R. Racke \& S. Zheng [45] study the problem of maximal $L^{p}$-regularity and asymptotic behavior of the solution and prove the existence of a global attractor to the non-viscous Cahn-Hilliard equation (that is, (1.1) with $\alpha=0$ ) with dynamic boundary conditions. Besides, the problem has also been analyzed as a dissipative dynamical system in $[\mathbf{4 1}]$, where the main result is the construction of a family of exponential attractors which is robust with respect to the viscosity coefficient $\alpha$. 
Another well-known mathematical model which describes the behavior of the phases, but in presence of temperature variations and in absence of mechanical stresses, is given by the Allen-Cahn equation (see [1]) suitably coupled with the heat equation. The resulting system governs the order parameter (or phase-field) and the temperature. Linearizing with respect to a suitable critical temperature at which the two phases coexist, one obtains the well-known phase-field system (see [7], cf. also [5])

$$
\begin{gathered}
\alpha \partial_{t} \phi-\Delta \phi+F_{1}^{\prime}(\phi)-\theta=0, \\
\partial_{t}(\varepsilon \theta+\phi)-\Delta \theta=0,
\end{gathered}
$$

in $\Omega \times(0,+\infty)$. Here $\psi$ is again the order parameter, $\theta$ denotes the (relative) temperature, $\alpha$ and $\varepsilon$ are given positive constants. The analysis of the dissipative dynamical system generated by equations like (1.3)-(1.4) equipped with rather standard boundary conditions (that is, Neumann or Dirichlet) has been done in a number of papers (see $[\mathbf{6}],[\mathbf{3}],[\mathbf{4}],[\mathbf{3 4}],[\mathbf{3 5}],[\mathbf{3 6}],[\mathbf{3 9}]$ ), proving theorems about existence of global and/or exponential attractors. Lately, the asymptotic behavior of single solutions has been investigated by means of the Łojasiewicz-Simon inequality (see $[\mathbf{2 9}],[\mathbf{3 0}]$ where singular potentials are considered, cf. also [53]). System (1.3)-(1.4) can be viewed as a singular perturbation of the celebrated Cahn-Hilliard equation that accounts for phase separation dynamics (see [12], cf. also [44] and references therein). In fact, if we formally set $\varepsilon=0$ in equation (1.4), then we can easily deduce the viscous Cahn-Hilliard equation (1.1) (see [43]) which reduces to the usual Cahn-Hilliard equation when $\alpha=0$. The rigorous links between system (1.3)-(1.4) and equation (1.1) have been studied by several authors. We refer the reader to $[\mathbf{4 2}]$ for more references. Moreover, in this paper, the authors construct a family of exponential attractors $\left\{\mathcal{M}_{\varepsilon}\right\}$ for a system like (1.3)-(1.4) with Dirichlet or Neumann boundary conditions for $\phi$ and $\theta$, and in the limit case, they obtain an exponential attractor $\mathcal{M}_{0}$ for (1.1). Then they prove its robustness, i.e., the Hausdorff distance between $\mathcal{M}_{\varepsilon}$ and $\mathcal{M}_{0}$ tends to 0 as $\varepsilon$ goes to 0 in an explicitly controlled way. The dynamic condition (1.2) can also be associated with system (1.3)-(1.4) (see $[\mathbf{9}],[\mathbf{2 9}],[\mathbf{2 3}],[\mathbf{2 4}]$ ). The authors in [9] prove well-posedness results as well as the convergence of the solution to a steady state by means of the Łojasiewicz-Simon technique. A more general analysis can be found in [23] and [29], where the problem is analyzed within the theory of dissipative dynamical systems. The authors in [29] show that the existence of the global attractor $\mathcal{A}_{\varepsilon}$ and its upper semicontinuity at $\varepsilon=0$. Moreover, they establish the existence of an exponential attractor. This fact, in particular, entails that $\mathcal{A}_{\varepsilon}$ has finite fractal dimension. Furthermore, in [23], we analyze a wider class of boundary conditions for $\theta$ and we allow $\theta$ to vary in a larger phase-space than the one in $[\mathbf{2 9}]$. Finally, in [24], we construct a robust family of exponential attractors with respect to $\varepsilon$ for problem (1.3)-(1.4) with dynamic boundary conditions like (1.2) and Neumann boundary conditions for $\theta$.

Our main goals are comparable with the ones cited in the papers above. We will consider a non-isothermal phase separation governed by the Cahn-Hilliard equation (1.1). The derivation is illustrated in [22], where we propose a non-isothermal version of (1.1) consisting of an evolution equation of fourth order for $\phi$ and an evolution equation of second order (similar to (1.4)) for the temperature function $\theta$. There, we also show how to derive all the boundary conditions (including dynamic 
for $\theta$ ) as part of energy and mass conservation laws. Our methods (see also [23]) are based on the derivation of dynamic boundary conditions in the context of heat and wave equations devised in [32].

Then, we are concerned with the following system of initial value problems:

$$
\begin{gathered}
\partial_{t} \phi=\Delta \mu, \text { for } x \in \Omega, \\
\mu=\alpha \partial_{t} \phi-\Delta \phi+F_{1}^{\prime}(\phi)-\delta \theta, \text { for } x \in \Omega, \\
\varepsilon \partial_{t} \theta-k_{2} \Delta \theta=-\delta \partial_{t} \phi-F_{3}^{\prime}(\theta), \text { for } x \in \Omega,
\end{gathered}
$$

subject to the boundary conditions

$$
\begin{gathered}
\partial_{n} \mu=0, \text { for } x \in \Gamma, \\
\partial_{t} \phi=\Delta_{\Gamma} \phi-\partial_{n} \phi-\phi-F_{2}^{\prime}(\phi), \text { for } x \in \Gamma, \\
b \partial_{n} \theta+c \theta=0, \text { for } x \in \Gamma,
\end{gathered}
$$

and initial conditions

$$
\phi_{\mid t=0}=\phi_{0}, \theta_{\mid t=0}=\theta_{0},
$$

where we distinguish the following cases: if $b>0, c \geq 0$ then (1.10) includes Neumann and Robin boundary conditions; if $b=0, c>0$ then (1.10) is a Dirichlet boundary condition. Here $k_{2}>0$ is the diffusion coefficient, $\delta>0$ is the latent heat parameter and $\varepsilon>0$. The functions $F_{i}(i=1,2,3)$ are quite general potential functions and $\Delta_{\Gamma}$ is the Laplace-Beltrami operator on the boundary $\Gamma$. In what follows, we shall also take $k_{2}=1$. Here, we would also like to mention the following papers in $[\mathbf{2}],[\mathbf{5}],[\mathbf{8}],[\mathbf{1 0}],[\mathbf{1 1}],[\mathbf{3 1}]$. In all these articles, the differential model describing non-isothermal phase separation is given by the system (1.5)-(1.7), when both $\theta$ and $\phi$ satisfy Dirichlet or Neumann boundary conditions. For the analysis on such systems or related problems (when memory effects are also incorporated in (1.5)$(1.7)$ ), we refer the reader to [31] where plenty of references are properly quoted. Well-posedness and maximal regularity, as well as asymptotic behavior for the nonviscous Cahn-Hilliard equation in the presence of temperature variations (that is, system (1.5)-(1.7) with $F_{2}=F_{3} \equiv 0$ ), equipped with linear dynamic boundary conditions for $\phi$ (of the form (1.2)) and Robin and Neumann boundary conditions for the temperature function $\theta$ (see (1.10)) was studied in [46]. There, the authors also prove convergence of solutions to steady states as $t \rightarrow+\infty$. Besides, employing classical methods, that is, fixed-point theorems and standard energy methods, we prove in $[\mathbf{2 2}]$ that the original system (1.5)-(1.11) is well-posed in a suitable Sobolev setting, by formulating appropriate approximate problems $P_{\varepsilon}$ and letting $\varepsilon \rightarrow 0^{+}$. However, in that paper, we are unable to prove that our problem generates a dynamical system, hence the existence of a nonlinear continuous semigroup does not follow. As far as we know, the system (1.5)-(1.11) has not yet been analyzed within the theory of infinite-dimensional dynamical theory. Thus, planning in doing so, we will also include nonlinear dynamic boundary conditions for $\phi$, as well as quite general nonlinearities $F_{1}, F_{2}, F_{3}$ in (1.6), (1.9), (1.7). Besides, we develop a Galerkin approximation scheme for a problem with dynamic boundary conditions by interpreting the boundary condition as a separate evolution equation on $\Gamma$. 
We assume throughout that the functions $F_{i}: \mathbf{R} \rightarrow \mathbf{R}, i=1,2,3$ are given $C^{2,1}$-functions satisfying the conditions

$$
\begin{gathered}
\lim _{|s| \rightarrow+\infty} \inf F_{i}^{\prime \prime}(s)>0, \text { for all } i=1,2,3, \\
F_{3}^{\prime}(s) s \geq-\zeta_{1}, \text { when } b, c>0 \text { and } b=0, c>0,
\end{gathered}
$$

and

$$
F_{3}^{\prime}(s) s \geq \zeta_{2} s^{2}-\zeta_{3}, \text { when } b>0, c=0,
$$

for some constants $\zeta_{1}, \zeta_{3} \geq 0$ and $\zeta_{2}>0$ and all real $s \in \mathbf{R}$.

Based on ideas from $[\mathbf{4 1}]$, we will prove existence and uniqueness of solutions to (1.5)-(1.11) based on a Faedo-Galerkin approximation scheme. It is worth mentioning that with respect to $[\mathbf{2 2}]$ and $[\mathbf{4 6}]$, there are some generalizations. In fact, our assumptions on the nonlinearities $F_{1}$ and $F_{2}$ are more general. Note that only the case $F_{2} \equiv 0, F_{3} \equiv 0$ and assuming that $F_{1} \in C^{4-}(\mathbf{R})$ has a polynomially controlled growth of degree six was discussed in [46]. Our main goal is to show that the above problem generates a dissipative dynamical system possessing a global attractor as well as an exponential attractor; however we do not analyze how they depend on $\varepsilon, \delta$ and $\alpha$. Thus it is interesting to note that if we formally take $\varepsilon=\delta=0$ in equations (1.6)-(1.7) and then taking $F_{3} \equiv 0$, then we can easily deduce the viscous $(\alpha>0)$ and non-viscous $(\alpha=0)$ Cahn-Hilliard equations (1.1) after suitable transformations. This issues are analyzed in $[\mathbf{2 7}]$ and $[\mathbf{2 8}]$. In particular, under suitable assumptions on these parameters, our main results in $[\mathbf{2 7}]$ allow us to show that the global attractor $\mathcal{A}_{0,0,0}$ of the non-viscous Cahn-Hilliard equation (corresponding to $(\varepsilon, \delta, \alpha)=(0,0,0))$ is upper semicontinuous at $(0,0,0)$ with respect to the family of global attractors $\mathcal{A}_{\varepsilon, \delta, \alpha}$. Finally, in [28], we construct a family of exponential attractors $\mathcal{M}_{\varepsilon, \delta, \alpha}$ which is a robust perturbation of an exponential attractor $\mathcal{M}_{0,0, \alpha}$ of the (isothermal) viscous $(\alpha>0)$ Cahn-Hilliard equation, namely, the symmetric Hausdorff distance between $\mathcal{M}_{\varepsilon, \delta, \alpha}$ and $\mathcal{M}_{0,0, \alpha}$ goes to 0 , for each fixed value of $\alpha>0$, as $(\varepsilon, \delta)$ goes to $(0,0)$, in an explicitly controlled way. Moreover, the robustness of this family of exponential attractors $\mathcal{M}_{\varepsilon, \delta, \alpha}$ with respect to $(\delta, \alpha) \rightarrow(0,0)$, for each fixed value of $\varepsilon>0$, is also obtained.

We outline the plan of the paper as follows. In Section 2 we recall some known facts about the Cahn-Hilliard equation (1.1) and introduce suitable phase spaces. Section 3 is devoted to the existence and uniqueness of solutions to problem (1.5)(1.11) and the derivation of suitable a priori estimates. The existence of a bounded absorbing set and, then, of the global attractor is the goal of Section 4 . The existence of an exponential attractor is demonstrated within the same section.

\section{Preliminary results}

In order to solve the system (1.5) - (1.11), we will derive a priori estimates for the solutions in suitable phase spaces. In order to give a rigorous formulation of our problem, we next set up our framework. We note that the system (1.5) - (1.11) possesses the following conservation law:

$$
\langle\phi(t)\rangle=\left\langle\phi_{0}\right\rangle:=M_{0},
$$

where $\langle\cdot\rangle$ denotes the average over $\Omega$. Let us also observe that if the value of $\phi(t)$ and $\theta(t)$ are known for some $t=T$, then the value of the chemical potential $\mu(T)$ 
can be found by solving the following boundary value problem:

$$
\begin{gathered}
\mu(T)-\alpha \Delta \mu(T)=-\Delta \phi(T)+F_{1}^{\prime}(\phi(T))-\delta \theta(T) \text { in } \Omega, \\
\partial_{n} \mu(T)=0 \text { on } \Gamma .
\end{gathered}
$$

Thus, it is only required to find the functions $\phi(t)$ and $\theta(t)$. In what follows, we shall take $\varepsilon=1$. Following [41], it is more convenient, however, to introduce the unknown function $\psi(t):=\phi(t)_{\mid \Gamma}$, defined on the boundary $\Gamma$ and to rewrite $(1.5)-(1.11)$ as

$$
\begin{gathered}
\partial_{t} \phi=\Delta \mu, \text { for } x \in \Omega, \\
\mu=\alpha \partial_{t} \phi-\Delta \phi+F_{1}^{\prime}(\phi)-\delta \theta, \text { for } x \in \Omega, \\
\partial_{t} \theta-\Delta \theta=-\delta \partial_{t} \phi-F_{3}^{\prime}(\theta), \text { for } x \in \Omega,
\end{gathered}
$$

subject to the boundary conditions

$$
\begin{gathered}
\partial_{n} \mu=0, \text { for } x \in \Gamma, \\
\partial_{t} \psi=\Delta_{\Gamma} \psi-\partial_{n} \phi-\psi-F_{2}^{\prime}(\psi), \text { for } x \in \Gamma, \\
b \partial_{n} \theta+c \theta=0, \text { for } x \in \Gamma,
\end{gathered}
$$

and initial conditions

$$
\phi_{\mid t=0}=\phi_{0}, \psi_{\mid t=0}=\psi_{0}, \theta_{\mid t=0}=\theta_{0}
$$

where $\psi_{0}=\phi_{0 \mid \Gamma}$.

The boundary condition (2.8) will be interpreted as an additional second-order parabolic equation on the boundary $\Gamma$. From now on, throughout the paper, we denote by $\|\cdot\|_{p}$ and $\|\cdot\|_{p, \Gamma}$, the norms on $L^{p}(\Omega)$ and $L^{p}(\Gamma)$, respectively. The inner products in $L^{p}(\Omega)$ and $L^{p}(\Gamma)$ will be denoted by $\langle\cdot, \cdot\rangle_{p}$ and $\langle\cdot, \cdot\rangle_{p, \Gamma}$, respectively. Also, the norms on $H^{s}(\Omega)$ and $H^{s}(\Gamma)$ are indicated by $\|\cdot\|_{H^{s}}$ and $\|\cdot\|_{H^{s}(\Gamma)}$, respectively. Any space $\mathbb{V}_{s}:=H^{s}(\Omega) \oplus H^{s}(\Gamma)(s \in \mathbf{N})$ is the completion of $C^{s}(\bar{\Omega})$ under the natural Sobolev norms. For example, for $s=0,1$, the spaces

$$
\mathbb{V}_{s}=\overline{C^{s}(\bar{\Omega})}\|\cdot\|_{\mathbb{V}_{s}},
$$

where the norms $\|\cdot\|_{\mathbb{V}_{s}}$ are given by

$$
\|(\phi, \psi)\|_{\mathbb{V}_{1}}^{2}=\int_{\Omega}|\nabla \phi|^{2} d x+\int_{\Gamma}\left|\nabla_{\Gamma} \psi\right|^{2} d S+\int_{\Gamma}|\psi|^{2} d S
$$

and

$$
\|(\phi, \psi)\|_{\mathbb{V}_{0}}^{2}=\int_{\Omega}|\phi|^{2} d x+\int_{\Gamma}|\psi|^{2} d S,
$$

respectively. It easy to see that we can identify $\mathbb{V}_{s}=H^{s}(\Omega) \times H^{s}(\Gamma)$ under these norms, when $s=0,1$. We have the embedding $H^{3 / 2}(\Omega) \subset \mathbb{V}_{1} \subset L^{2}(\Omega)$. As we mentioned in the introduction, we distinguish between three cases: $(i) b>0$, $c>0$; (ii) $b>0, c \geq 0$; (iii) $b=0, c>0$. For this, let us define the family of operators $A_{K}:=-\Delta$ on the Banach space $L^{2}(\Omega)$, when $K \in\{R, N, D\}$ and $R, N$ 
and $D$ stand for Robin, Neumann, and Dirichlet boundary conditions, respectively. Furthermore, let

$$
\begin{gathered}
\mathcal{D}\left(A_{D}\right)=\left\{\theta \in H^{2}(\Omega) \mid \theta=0 \text { on } \Gamma\right\}, \\
\mathcal{D}\left(A_{N}\right)=\left\{\theta \in H^{2}(\Omega) \mid \partial_{n} \theta=0 \text { on } \Gamma\right\}, \\
\mathcal{D}\left(A_{R}\right)=\left\{\theta \in H^{2}(\Omega) \mid b \partial_{n} \theta+c \theta=0 \text { on } \Gamma\right\}, \text { if } b>0, c \geq 0 .
\end{gathered}
$$

Then, it is well known that $A_{K}$, when $K=D, N, R$, generates a bounded analytic semigroup $e^{-A_{K} t}$ on $L^{2}(\Omega)$. Also, each $A_{K}$ is nonnegative and self-adjoint on $L^{2}(\Omega)$. Note that $\mathcal{D}\left(A_{K}\right)$ is densely contained in $L^{2}(\Omega)$, for $K \in\{R, N, D\}$. Let us also recall that $A_{N}^{-1}: L_{0}^{2}(\Omega) \rightarrow L_{0}^{2}(\Omega)$, where $L_{0}^{2}(\Omega)=L^{2}(\Omega) \cap\{\langle\phi\rangle=0\}$, is a well defined operator and is usually referred to as the inverse Laplacian with Neumann boundary conditions. Henceforth, we will always refer to the following norm in $H^{-1}(\Omega)=\left(H_{N}^{1}(\Omega)\right)^{*}$, equivalent to the standard one as follows:

$$
\|u\|_{H^{-1}}^{2}=\left\|A_{N}^{-1 / 2}(u-\langle u\rangle)\right\|_{2}^{2}+\langle u\rangle^{2} .
$$

Having established this framework, we introduce the phase space (cf. also [16], [41]) for our problem (2.4) - (2.10):

$\mathbb{Y}_{K}^{\alpha}:=\left\{\Phi=(\phi, \psi, \theta) \in H^{2}(\Omega) \times H^{2}(\Gamma) \times \mathcal{D}\left(A_{K}\right): \mu \in H^{1}(\Omega), \sqrt{\alpha} \mu \in H^{2}(\Omega)\right.$,

$$
\left.\phi_{\mid \Gamma}=\psi,\left(\partial_{n} \mu\right)_{\mid \Gamma}=0\right\}, K \in\{D, N, R\},
$$

with the obvious norm

$$
\begin{aligned}
& \|\Phi\|_{\mathbb{Y}_{K}^{\alpha}}^{2}:=\|(\phi, \psi)\|_{\mathbb{V}_{2}}^{2}+\|\mu\|_{H^{1}}^{2}+\alpha\|\mu\|_{H^{2}}^{2}+\|\theta\|_{H^{2}}^{2} \\
& =\|\phi\|_{H^{2}}^{2}+\|\psi\|_{H^{2}(\Gamma)}^{2}+\|\mu\|_{H^{1}}^{2}+\alpha\|\mu\|_{H^{2}}^{2}+\|\theta\|_{H^{2}}^{2} .
\end{aligned}
$$

DeFinition 1. Let us consider $T>0$ fixed, but otherwise arbitrary and let $K \in\{D, N, R\}$. By a solution of $(2.4)-(2.10)$ we mean a triplet of functions

$$
(\phi(t), \psi(t), \theta(t)) \in L^{\infty}\left([0, T], \mathbb{Y}_{K}^{\alpha}\right)
$$

with

$$
\partial_{t} \phi(t) \in L^{2}\left([0, T], H^{1}(\Omega)\right), \partial_{t} \psi(t) \in L^{2}\left([0, T], H^{1}(\Gamma)\right)
$$

and

$$
\partial_{t} \theta(t) \in L^{2}\left([0, T], L^{2}(\Omega)\right)
$$

which satisfy the equations in the sense of equalities in the spaces $L^{2}\left([0, T], L^{2}(\Omega)\right)$ and $L^{2}\left([0, T], L^{2}(\Gamma)\right)$. Moreover, since $\Omega \subset \mathbf{R}^{3}$, we have the embeddings $H^{2}(\Omega) \subset$ $L^{\infty}(\Omega), H^{2}(\Gamma) \subset L^{\infty}(\Gamma)$, therefore the nonlinearities $F_{i}(i=1,2,3)$ are well defined and belong to the space $C\left([0, T], L^{2}(\Omega)\right)$ and $C\left([0, T], L^{2}(\Gamma)\right)$. Also, by regularity theory, we obtain $\mu(t) \in L^{2}\left([0, T], H^{3}(\Omega)\right)$ and thus the boundary conditions $(2.7)-(2.9)$ are well defined.

We close this section with the definition of the weaker energy space $\mathbb{X}:=$ $\mathbb{V}_{1} \times L^{2}(\Omega)$ for our problem (2.4) - (2.10) through the norm given by

$$
\begin{gathered}
\|\Phi\|_{\mathbb{X}}^{2}=\|(\phi, \psi)\|_{\mathbb{V}_{1}}^{2}+\|\theta\|_{2}^{2} \\
=\|\phi\|_{H^{1}}^{2}+\|\psi\|_{H^{1}(\Gamma)}^{2}+\|\theta\|_{2}^{2},
\end{gathered}
$$


for $\Phi=(\phi, \psi, \theta)$. Note that $\mathbb{Y}_{K}^{\alpha}$ is continuously embedded and dense in $\mathbb{X}$ for each $K \in\{D, N, R\}$. For our convenience, we also set

$$
Z_{D}:=H_{0}^{1}(\Omega), \quad Z_{K}:=H^{1}(\Omega), \quad \text { if } K \in\{N, R\} .
$$

\section{Uniform a priori estimates and existence of solutions}

In this section, we derive several estimates for the solutions of the problem $(2.4)-(2.10)$ which are necessary for the study of the asymptotic behavior. In the first step, we obtain dissipative estimates for solutions in the spaces $\mathbb{X}$ and $\mathbb{Y}_{K}^{\alpha}$. Throughout the entire section $K \in\{D, N, R\}$ is fixed.

For convenience, we rewrite $(2.4)-(2.10)$ as the following system of equations:

$$
\begin{gathered}
\left\{\begin{array}{c}
\left(\alpha+A_{N}^{-1}\right) \partial_{t} \phi-\Delta \phi=\delta \theta-F_{1}^{\prime}(\phi)+\langle\mu\rangle \text { in } \Omega \times(0, \infty), \\
\partial_{t} \psi=\Delta_{\Gamma} \psi-\partial_{n} \phi-\psi-F_{2}^{\prime}(\psi) \text { on } \Gamma \times(0, \infty), \phi_{\mid \Gamma}=\psi
\end{array}\right. \\
\left\{\begin{array}{c}
\partial_{t} \theta-\Delta \theta=-\delta \partial_{t} \phi-F_{3}^{\prime}(\theta) \text { in } \Omega \times(0, \infty), \\
b \partial_{n} \theta+c \theta=0, \text { for } x \in \Gamma \times(0, \infty),
\end{array}\right.
\end{gathered}
$$

and

$$
\phi_{\mid t=0}=\phi_{0}, \psi_{\mid t=0}=\psi_{0}, \theta_{\mid t=0}=\theta_{0} .
$$

Recall that $\mu$ can be found from the linear elliptic problem (2.2) $-(2.3)$ when both $\phi$ and $\theta$ are known. Moreover, from equations (2.4)-(2.5), we have $\mu=\langle\mu\rangle-A_{N}^{-1}\left(\partial_{t} \phi\right)$, where $\langle\mu\rangle=-\langle\Delta \phi\rangle+\left\langle F_{1}^{\prime}(\phi)\right\rangle-\delta\langle\theta\rangle$, since $\left\langle\partial_{t} \phi\right\rangle=0$, due to the mass conservation (2.1). We will come back to (3.1)-(3.3), in the last part of this section, when we will show existence of solutions based on a Faedo-Galerkin approximation scheme.

Proposition 2. Let the nonlinearities $F_{i}(i=1,2,3)$ satisfy $(1.12)-(1.14)$ and let $(\phi(t), \psi(t), \theta(t))$ be a sufficiently regular solution of (2.4)-(2.10) or (3.1)-(3.3). Then, the following estimate holds:

$$
\begin{gathered}
\|(\phi(t), \psi(t), \theta(t))\|_{\mathbb{X}}^{2}+\left\langle F_{1}(\phi(t)), 1\right\rangle_{2}+\left\langle F_{2}(\psi(t)), 1\right\rangle_{2, \Gamma} \\
+\int_{t}^{t+1}\left(\left\|F_{1}(\phi(s))\right\|_{1}+\left\|F_{2}(\psi(s))\right\|_{1, \Gamma}+\left\|\partial_{t} \phi(s)\right\|_{H^{-1}}^{2}\right) d s \\
+\int_{t}^{t+1}\left(\alpha\left\|\partial_{t} \phi(s)\right\|_{2}^{2}+\left\|\partial_{t} \psi(s)\right\|_{2, \Gamma}^{2}+\|\nabla \theta(s)\|_{2}^{2}+\frac{c}{b}\|\theta(s)\|_{2, \Gamma}^{2}\right) d s \\
(3.4) \leq Q\left(\|(\phi(0), \psi(0), \theta(0))\|_{\mathbb{X}}^{2}+\left\langle F_{1}(\phi(0)), 1\right\rangle_{2}+\left\langle F_{2}(\psi(0)), 1\right\rangle_{2, \Gamma}\right) e^{-\rho t}+C,
\end{gathered}
$$

where the positive constants $\rho, C$ and the monotonic function $Q$ are independent of $\alpha$ and $t$. 
Proof. Multiplying equation (2.5), (2.6), (2.8) scalarly by $\partial_{t} \phi, \theta$, and $\partial_{t} \psi$, respectively, then (2.4) by $A_{N}^{-1}\left(\partial_{t} \phi\right)$, integrating over $\Omega$ using the boundary conditions and summing up over the relations that we obtain, we deduce

$$
\begin{gathered}
\frac{d}{d t}\left[\|\nabla \phi(t)\|_{2}^{2}+\left\|\nabla_{\Gamma} \psi(t)\right\|_{2, \Gamma}^{2}+\|\psi(t)\|_{2, \Gamma}^{2}+\|\theta(t)\|_{2}^{2}\right. \\
\left.+2\left\langle F_{1}(\phi(t)), 1\right\rangle_{2}+2\left\langle F_{2}(\psi(t)), 1\right\rangle_{2, \Gamma}\right] \\
+2\left\|\partial_{t} \phi(t)\right\|_{H^{-1}}^{2}+2 \alpha\left\|\partial_{t} \phi(t)\right\|_{2}^{2} \\
+2\left\|\partial_{t} \psi(t)\right\|_{2, \Gamma}^{2}+2\left\langle A_{K} \theta(t), \theta(t)\right\rangle_{2}+2\left\langle F_{3}^{\prime}(\theta(t)), \theta(t)\right\rangle_{2}=0,
\end{gathered}
$$

since $\left\langle\langle\mu(t)\rangle, \partial_{t} \phi(t)\right\rangle_{2}=|\Omega|\langle\mu(t)\rangle\left\langle\partial_{t} \phi(t)\right\rangle=0$, because of (2.1). Here $|\Omega|$ denotes the volume of $\Omega$. Let us now introduce the functions $\bar{\phi}(t):=\phi(t)-M_{0}$ and $\bar{\psi}(t):=$ $\psi(t)-M_{0}$ and note that $\langle\bar{\phi}(t)\rangle=0$. Then take the inner product of equations $(2.5),(2.4)$ and $(2.8)$ with $2 \xi \bar{\phi}(t), 2 \xi A_{N}^{-1} \bar{\phi}(t)$ and $2 \xi \bar{\psi}(t)$ in $L^{2}(\Omega)$ and $L^{2}(\Gamma)$, respectively, and recall that $\mu=\langle\mu\rangle-A_{N}^{-1}\left(\partial_{t} \phi\right)$. We deduce that

$$
\begin{gathered}
\frac{d}{d t}\left[\alpha \xi\|\bar{\phi}(t)\|_{2}^{2}+\xi\|\bar{\psi}(t)\|_{2, \Gamma}^{2}+\xi\|\bar{\phi}(t)\|_{H^{-1}}^{2}\right] \\
+2 \xi\left[\|\nabla \bar{\phi}(t)\|_{2}^{2}+\left\|\nabla_{\Gamma} \bar{\psi}(t)\right\|_{2, \Gamma}^{2}+\langle\psi(t), \bar{\psi}(t)\rangle_{2, \Gamma}\right] \\
+2 \xi\left[\left\langle F_{1}^{\prime}(\phi(t)), \bar{\phi}(t)\right\rangle_{2}+\left\langle F_{2}^{\prime}(\psi(t)), \bar{\psi}(t)\right\rangle_{2, \Gamma}\right] \\
=2 \delta \xi\langle\bar{\phi}(t), \theta(t)\rangle_{2}+2 \xi\langle\bar{\phi}(t),\langle\mu(t)\rangle\rangle_{2} .
\end{gathered}
$$

Summing up the relations (3.5) and (3.6), we obtain

$$
\frac{d}{d t} E(t)+\tau E(t)=h_{1}(t),
$$

where $0<\tau<\xi$ is sufficiently small and

$$
\begin{aligned}
E(t) & =\|\nabla \phi(t)\|_{2}^{2}+\left\|\nabla_{\Gamma} \psi(t)\right\|_{2, \Gamma}^{2}+\|\psi(t)\|_{2, \Gamma}^{2}+\|\theta(t)\|_{2}^{2}+2\left\langle F_{1}(\phi(t)), 1\right\rangle_{2} \\
& +2\left\langle F_{2}(\psi(t)), 1\right\rangle_{2, \Gamma}+\alpha \xi\|\bar{\phi}(t)\|_{2}^{2}+\xi\|\bar{\psi}(t)\|_{2, \Gamma}^{2}+\xi\|\bar{\phi}(t)\|_{H^{-1}}^{2} .
\end{aligned}
$$

The function $h_{1}$ is given by

$$
\begin{gathered}
h_{1}(t)=-2\left(\left\|\partial_{t} \phi(t)\right\|_{H^{-1}}^{2}+\alpha\left\|\partial_{t} \phi(t)\right\|_{2}^{2}+\left\|\partial_{t} \psi(t)\right\|_{2, \Gamma}^{2}\right)-2\left\langle A_{K} \theta(t), \theta(t)\right\rangle_{2} \\
-2 \tau\left[\left\langle F_{1}^{\prime}(\phi(t)) \bar{\phi}(t)-F_{1}(\phi(t)), 1\right\rangle_{2}+\left\langle F_{2}^{\prime}(\psi(t)) \bar{\psi}(t)-F_{2}(\psi(t)), 1\right\rangle_{2, \Gamma}\right] \\
-2\left\langle F_{3}^{\prime}(\theta(t)), \theta(t)\right\rangle_{2}-2(\xi-\tau)\left[\left\langle F_{1}^{\prime}(\phi(t)), \bar{\phi}(t)\right\rangle_{2}+\left\langle F_{2}^{\prime}(\psi(t)), \bar{\psi}(t)\right\rangle_{2, \Gamma}\right] \\
-(2 \xi-\tau)\left(\|\nabla \bar{\phi}(t)\|_{2}^{2}+\left\|\nabla_{\Gamma} \bar{\psi}(t)\right\|_{2, \Gamma}^{2}\right)+\left(\tau\|\psi(t)\|_{2, \Gamma}^{2}-2 \xi\langle\psi(t), \bar{\psi}(t)\rangle_{2, \Gamma}\right) \\
+\tau\left[\alpha \xi\|\bar{\phi}(t)\|_{2}^{2}+\xi\|\bar{\psi}(t)\|_{2, \Gamma}^{2}+\xi\|\bar{\phi}(t)\|_{H^{-1}}^{2}\right] \\
+2 \delta \xi\langle\bar{\phi}(t), \theta(t)\rangle_{2}+2 \xi\langle\bar{\phi}(t),\langle\mu(t)\rangle\rangle_{2}+\tau\|\theta(t)\|_{2}^{2} .
\end{gathered}
$$

First, it easy to see that

$$
2 \xi\langle\bar{\phi}(t),\langle\mu(t)\rangle\rangle_{2}=2 \xi\langle\mu(t)\rangle\langle\bar{\phi}(t)\rangle=0
$$


Holder and Young inequalities yield

$$
\tau\|\psi(t)\|_{2, \Gamma}^{2}-2 \xi\langle\psi(t), \bar{\psi}(t)\rangle_{2, \Gamma} \leq-(\xi-2 \tau)\|\bar{\psi}(t)\|_{2, \Gamma}^{2}+C_{M_{0}},
$$

where $C_{M_{0}}>0$ depends obviously on $M_{0}, \xi, \tau$ and $|\Omega|,|\Gamma|$. Here, $|\Gamma|$ denotes the measure of $\Gamma$. We emphasize that when $K=D,\left\langle A_{D} \theta(t), \theta(t)\right\rangle_{2}$ is equivalent to the norm in $H_{0}^{1}(\Omega)$, while in the case $K=R,\left\langle A_{R} \theta(t), \theta(t)\right\rangle_{2}$ is equivalent to a norm in $H^{1}(\Omega)$. The case $K=N$ is more delicate since $\left\langle A_{N} \theta(t), \theta(t)\right\rangle_{2}=\|\nabla \theta\|_{2}^{2}$. In this case, we will need assumption (1.14) in order to control the $H^{1}-$ norm of the temperature function $\theta$. It remains to also note that due to assumption (1.12), we have for $i=1,2$ :

$$
\begin{gathered}
F_{i}(s)-C_{F_{i}, M_{0}} \leq C_{i}\left(s-M_{0}\right)^{2}+F_{i}^{\prime}(s)\left(s-M_{0}\right), \forall s \in \mathbf{R}, \\
\left|F_{i}^{\prime}(s)\right|(1+|s|) \leq 2 F_{i}^{\prime}(s)\left(s-M_{0}\right)+C_{F_{i}, M_{0}}^{\prime}, \forall s \in \mathbf{R},
\end{gathered}
$$

where $C_{i}, C_{F_{i}, M_{0}}$ and $C_{F_{i}, M_{0}}^{\prime}$ are sufficiently large constants. Consequently, using (3.9), (3.10), the obvious inequality $\|\bar{\phi}(t)\|_{H^{-1}}^{2} \leq\|\bar{\phi}(t)\|_{2}^{2} \leq C\|\nabla \bar{\phi}(t)\|_{2}^{2}$ (since $\langle\bar{\phi}(t)\rangle=0)$ and the assumptions $(1.13)-(1.14)$, it is possible to estimate $h_{1}$ as follows:

$$
\begin{gathered}
h_{1}(t) \leq-\left(2 C_{1}-\tau-C_{\Omega} \delta^{2} \xi\right)\|\theta\|_{Z_{K}}^{2} \\
-2\left(\left\|\partial_{t} \phi(t)\right\|_{H^{-1}}^{2}+\alpha\left\|\partial_{t} \phi(t)\right\|_{2}^{2}+\left\|\partial_{t} \psi(t)\right\|_{2, \Gamma}^{2}\right) \\
-(\xi-\tau)\left(\left\langle\left|F_{1}^{\prime}(\phi(t))\right|, 1+|\phi(t)|\right\rangle_{2}+\left\langle\left|F_{2}^{\prime}(\psi(t))\right|, 1+|\psi(t)|\right\rangle_{2, \Gamma}\right) \\
-\left[\xi-\tau-C_{2} \tau\left(\xi+C_{3}\right)-C_{4} \alpha \xi \tau\right]\left(\|\nabla \bar{\phi}(t)\|_{2}^{2}+\|\nabla \bar{\psi}(t)\|_{2, \Gamma}^{2}\right) \\
-\left(\xi-2 \tau\left(1+C_{5}\right)-\tau \xi\right)\|\bar{\psi}(t)\|_{2, \Gamma}^{2}+Q^{*}\left(M_{0}\right),
\end{gathered}
$$

where all the positive constants and the function $Q^{*}$ are independent of $t$. Here, recall again that $Z_{K}=H^{1}(\Omega)$ when $K \in\{N, R\}$ and $Z_{D}=H_{0}^{1}(\Omega)$. It follows that we can fix the parameters $\xi$ and $\tau$ such that

$$
\begin{gathered}
\frac{d}{d t} E(t)+\tau_{1} E(t)+\tau_{2}\left(\|\nabla \bar{\phi}(t)\|_{2}^{2}+\|\nabla \bar{\psi}(t)\|_{2, \Gamma}^{2}+\|\bar{\psi}(t)\|_{2, \Gamma}^{2}+\|\theta(t)\|_{Z_{K}}^{2}\right) \\
+\tau_{2}\left(\left\langle\left|F_{1}^{\prime}(\phi(t))\right|, 1+|\phi(t)|\right\rangle_{2}+\left\langle\left|F_{2}^{\prime}(\psi(t))\right|, 1+|\psi(t)|\right\rangle_{2, \Gamma}\right) \\
+2\left(\left\|\partial_{t} \phi(t)\right\|_{H^{-1}}^{2}+\alpha\left\|\partial_{t} \phi(t)\right\|_{2}^{2}+\left\|\partial_{t} \psi(t)\right\|_{2, \Gamma}^{2}\right) \leq C_{*},
\end{gathered}
$$

where the positive constants $\tau_{1}, \tau_{2}$ and $C_{*}$ are independent of $\alpha$ and $t$. However, note that $C_{*}$ depends on $M_{0}$. Applying now a suitable version of Gronwall's inequality to relation (3.11), using (3.8) and observing that the assumption (1.12) also implies that $\left|F_{i}(s)\right|-C \leq\left|F_{i}^{\prime}(s)\right|(1+|s|)$, for some positive constant $C$ and all $s \in \mathbf{R}$ (since the functions $F_{i}$ are monotonic if $|s|$ is large enough), we easily obtain estimate (3.4). This completes the proof of Proposition 2. 
Proposition 3. Let the assumptions of Proposition 2 hold. Then the following estimate holds for a regular solution $(\phi(t), \psi(t), \theta(t))$ of $(2.4)-(2.10)$ :

$$
\begin{gathered}
\alpha\left\|\partial_{t} \phi(t)\right\|_{2}^{2}+\left\|\partial_{t} \phi(t)\right\|_{H^{-1}}^{2}+\left\|\partial_{t} \psi(t)\right\|_{2, \Gamma}^{2} \\
+\|\theta(t)\|_{Z_{K}}^{2}+\int_{t}^{t+1}\left(\left\|\left(\partial_{t} \phi(s), \partial_{t} \psi(s)\right)\right\|_{\mathbb{V}_{1}}^{2}+\left\|\partial_{t} \theta(s)\right\|_{2}^{2}\right) d s \\
\leq Q\left(\|(\phi(0), \psi(0), \theta(0))\|_{\mathbb{Y}_{K}^{\alpha}}^{2}\right) e^{-\eta t}+C,
\end{gathered}
$$

for each $t \geq 0$, where the monotonic function $Q$ and the constants $\eta, C$ are independent of $t, \alpha$.

Proof. We give a formal derivation of (3.12), which can be justified within a Faedo-Galerkin approximation scheme (see the end of Section 3). Therefore, without loss of generality, we can (and do) differentiate (2.4), (2.5), (2.7), (2.8) and define

$$
(u(t), v(t), \varpi(t), m(t)):=\left(\partial_{t} \phi(t), \partial_{t} \psi(t), \partial_{t} \theta(t), \partial_{t} \mu(t)\right) .
$$

Then, we have

$$
\left\{\begin{array}{c}
\partial_{t} u(t)=\Delta m(t) \text { in } \Omega, \partial_{n} m(t)=0 \text { on } \Gamma \\
m(t)=\alpha \partial_{t} u(t)-\Delta u(t)+F_{1}^{\prime \prime}(\phi(t)) u(t)-\delta \varpi(t) \text { in } \Omega \\
\partial_{t} v(t)=\Delta_{\Gamma} v(t)-\partial_{n} u(t)-v(t)-F_{2}^{\prime \prime}(\psi) v \text { on } \Gamma, u_{\mid \Gamma}=v
\end{array}\right.
$$

Multiplying equation (2.6) scalarly by $\varpi(t)$ and integrating over $\Omega$, using the boundary condition, it follows that we have

$$
\frac{1}{2} \frac{d}{d t}\left[\left\langle A_{K} \theta(t), \theta(t)\right\rangle_{2}+2\left\langle F_{3}(\theta(t)), 1\right\rangle_{2}\right]+\|\varpi(t)\|_{2}^{2}=-\delta\langle u(t), \varpi(t)\rangle_{2} .
$$

Next, multiply the first, third and fourth equations of (3.13) scalarly by $A_{N}^{-1} u(t)$, $u(t)$ and $v(t)$, respectively, then integrate over $\Omega$, using the fact that $\langle u(t)\rangle=0$. Combining the resulting equation with (3.14), we deduce

$$
\begin{gathered}
\frac{1}{2} \frac{d}{d t}\left[\alpha\|u(t)\|_{2}^{2}+\|u(t)\|_{H^{-1}}^{2}+\|v(t)\|_{2, \Gamma}^{2}+\left\langle A_{K} \theta(t), \theta(t)\right\rangle_{2}+2\left\langle F_{3}(\theta(t)), 1\right\rangle_{2}\right] \\
+\|\nabla u(t)\|_{2}^{2}+\left\|\nabla_{\Gamma} v(t)\right\|_{2, \Gamma}^{2}+\|v(t)\|_{2, \Gamma}^{2}+\|\varpi(t)\|_{2}^{2}+\|\theta(t)\|_{Z_{K}}^{2} \\
=-\left\langle F_{1}^{\prime \prime}(\phi(t)), u^{2}\right\rangle_{2}-\left\langle F_{2}^{\prime \prime}(\psi(t)), v^{2}\right\rangle_{2, \Gamma}+\|\theta(t)\|_{Z_{K}}^{2}:=h_{2}(t) .
\end{gathered}
$$

Due to the assumption (1.12), we have $F_{i}^{\prime \prime}(s) \geq-C_{i}$, for $s \in \mathbf{R}$ and some positive constants $C_{i}$. Thus, we will estimate $h_{2}(t), t \geq 0$, using a standard Sobolev inequality (that is, $\|u\|_{2}^{2} \leq C_{1}\|u\|_{H^{-1}}\|\nabla u\|_{2}$, since $\langle u(t)\rangle=0$ ) as follows:

$$
\begin{gathered}
h_{2}(t) \leq C_{1}\|u(t)\|_{2}^{2}+C_{2}\|v(t)\|_{2, \Gamma}^{2}+\|\theta(t)\|_{Z_{K}}^{2} \\
\leq \eta\|\nabla u(t)\|_{2}^{2}+C_{\eta}\|u(t)\|_{H^{-1}}^{2}+C_{2}\|v(t)\|_{2, \Gamma}^{2}+\|\theta(t)\|_{Z_{K}}^{2},
\end{gathered}
$$


for a sufficiently small $\eta>0$ and large constant $C_{\eta}>0$. Therefore, choosing $\eta<1$ small, and using estimate (3.4), the embeddings $H^{2}(\Omega) \subset L^{\infty}(\Omega), H^{2}(\Gamma) \subset$ $L^{\infty}(\Gamma)$ imply that

$$
\sup _{t \geq 0} \int_{t}^{t+1} h_{2}(s) d s \leq Q_{1}\left(\|(\phi(0), \psi(0), \theta(0))\|_{\mathbb{Y}_{K}^{\alpha}}^{2}\right) e^{-\rho t}+C_{3},
$$

for some monotone increasing function $Q_{1}$ and positive constant $C_{3}$ that are independent of $t, \alpha$. Applying now a suitable version of Gronwall's inequality (see e.g., [26, Lemma 2.5]) to relation (3.15), using estimate (3.17), we deduce

$$
\begin{gathered}
\alpha\|u(t)\|_{2}^{2}+\|u(t)\|_{H^{-1}}^{2}+\|v(t)\|_{2, \Gamma}^{2}+\left\langle A_{K} \theta(t), \theta(t)\right\rangle_{2} \\
+2\left\langle F_{3}(\theta(t)), 1\right\rangle_{2}+\int_{t}^{t+1}\left[\|u(s)\|_{H^{1}}^{2}+\|v(s)\|_{H^{1}(\Gamma)}^{2}+\|\varpi(s)\|_{2}^{2}\right] d s \\
\leq Q_{2}\left(\alpha\|u(0)\|_{2}^{2}+\|u(0)\|_{H^{-1}}^{2}+\|v(0)\|_{2, \Gamma}^{2}+\|\theta(0)\|_{H^{2}}^{2}\right) e^{-\rho t}+C_{4},
\end{gathered}
$$

for a suitable positive constant $C_{4}$ and monotonic functions $Q_{1}, Q_{2}$ which are independent of $t$. Moreover, from (2.5), (2.8), we have

$$
\begin{gathered}
\alpha u(0)=\Delta \phi(0)-F_{1}^{\prime}(\phi(0))+\delta \theta(0)+\mu(0) \text { in } \Omega, \\
v(0)=\Delta_{\Gamma} \psi(0)-\partial_{n} \phi(0)-\psi(0)-F_{2}^{\prime}(\psi(0)) \text { on } \Gamma .
\end{gathered}
$$

Therefore, we deduce from $H^{2}(\Omega) \subset L^{\infty}(\Omega), H^{2}(\Gamma) \subset L^{\infty}(\Gamma)$, and the fact that $u(0)=\Delta \mu(0)$, that

$$
\begin{gathered}
\alpha\|u(0)\|_{2}^{2}+\|u(0)\|_{H^{-1}}^{2}+\|v(0)\|_{2, \Gamma}^{2} \\
\leq C_{5}\left(\|(\phi(0), \psi(0))\|_{\mathbb{V}_{2}}^{2}+\alpha\|\Delta \mu(0)\|_{2}^{2}+\|\nabla \mu(0)\|_{2}^{2}\right),
\end{gathered}
$$

where $C_{5}>0$ is independent of $t$ and $\alpha$. Finally, collecting estimates (3.4), (3.18) and taking (3.19) into account, we easily obtain our conclusion, for every $K \in$ $\{D, N, R\}$. The proof of Proposition 3 is complete.

We are now ready to give the dissipative estimate for solutions of our problem in the space $\mathbb{Y}_{K}^{\alpha}, K \in\{D, N, R\}$.

TheOrem 4. Let the assumptions of Proposition 1 hold. Then every solution $(\phi, \psi, \theta)$ of $(2.4)-(2.10)$ satisfies the estimate:

$$
\begin{gathered}
\|(\phi(t), \psi(t), \theta(t))\|_{\mathbb{Y}_{K}^{\alpha}}^{2}+\left\|\left(\partial_{t} \phi(t), \partial_{t} \psi(t)\right)\right\|_{\mathbb{V}_{0}}^{2}+\left\|\partial_{t} \theta(t)\right\|_{2}^{2} \\
\quad+\int_{t}^{t+1}\left\|\left(\partial_{t} \phi(s), \partial_{t} \psi(s), \partial_{t} \theta(s)\right)\right\|_{\mathbb{X}}^{2} d s \\
\leq Q_{\alpha}\left(\|(\phi(0), \psi(0), \theta(0))\|_{\mathbb{Y}_{K}^{\alpha}}^{2}\right) e^{-\rho_{1} t}+Q_{0}^{\alpha}\left(M_{0}\right),
\end{gathered}
$$

for every $t \geq 0$, where the monotonic functions $Q_{\alpha}, Q_{0}^{\alpha}$ and $\rho_{1}$ are independent of $t$, but they depend on $\alpha$. Moreover, the function $Q_{0}^{\alpha}$ depends on $M_{0}$. 
Proof. Recall that $\partial_{t} \phi=\Delta \mu$. Consequently, (3.18) can be also rewritten as

$$
\begin{gathered}
\alpha\|\Delta \mu(t)\|_{2}^{2}+\|\nabla \mu(t)\|_{2}^{2}+\left\|\partial_{t} \psi(t)\right\|_{2, \Gamma}^{2}+\|\theta(t)\|_{Z_{K}}^{2} \\
\leq Q_{2}\left(\alpha\|\Delta \mu(0)\|_{2}^{2}+\|\nabla \mu(0)\|_{2}^{2}+\left\|\partial_{t} \psi(0)\right\|_{2, \Gamma}^{2}+\|\theta(0)\|_{H^{2}}^{2}\right) e^{-\rho t}+C_{4} .
\end{gathered}
$$

Thus, in order to obtain the $H^{1}$ estimate for the function $\mu$, we need to get $\mu-$ part of estimate (3.20). To this end, we can estimate the average of $\mu(t)$ over $\Omega$ from $(2.5)$, as follows (note that $\left\langle\partial_{t} \phi(t)\right\rangle=0$ ):

$$
\begin{gathered}
\langle\mu(t)\rangle=-\langle\Delta \phi(t)\rangle+\left\langle F_{1}^{\prime}(\phi(t))\right\rangle-\delta\langle\theta(t)\rangle \\
=-\frac{|\Gamma|}{|\Omega|}\left\langle\partial_{n} \phi(t)\right\rangle_{\Gamma}+\left\langle F_{1}^{\prime}(\phi(t))\right\rangle-\delta\langle\theta(t)\rangle \\
=\frac{|\Gamma|}{|\Omega|}\langle\psi(t)\rangle_{\Gamma}+\frac{|\Gamma|}{|\Omega|}\left\langle F_{2}^{\prime}(\psi(t))\right\rangle_{\Gamma}+\left\langle F_{1}^{\prime}(\phi(t))\right\rangle-\delta\langle\theta(t)\rangle+\frac{|\Gamma|}{|\Omega|}\left\langle\partial_{t} \psi(t)\right\rangle_{\Gamma},
\end{gathered}
$$

where $\langle\cdot\rangle_{\Gamma}$ denotes the the total average over the boundary $\Gamma$. Also recall that $\langle\phi(t)\rangle=\left\langle\phi_{0}\right\rangle=M_{0}$. Since the terms $\langle\theta(t)\rangle$ and $\left\langle\partial_{t} \psi(t)\right\rangle_{\Gamma}$ are already estimated in (3.12), we only need to estimate the average of $\psi, F_{1}^{\prime}(\phi)$ and $F_{2}^{\prime}(\psi)$. To do so, recall that $\bar{\phi}(t):=\phi(t)-M_{0}$ and $\bar{\psi}(t):=\psi(t)-M_{0}$ and notice that we have $\langle\bar{\phi}(t)\rangle=0$. Then, multiplying the expression for $\mu$ in $(2.5)$ by $\bar{\phi}(t)$, and integrating over $\Omega$, we have after standard transformations,

$$
\begin{gathered}
\|\nabla \phi(t)\|_{2}^{2}+\left\|\nabla_{\Gamma} \psi(t)\right\|_{2, \Gamma}^{2}+\left\langle F_{1}^{\prime}(\phi(t)), \bar{\phi}(t)\right\rangle_{2}+\left\langle F_{2}^{\prime}(\psi(t)), \bar{\psi}(t)\right\rangle_{2, \Gamma} \\
+\left\langle\psi(t), \psi(t)-M_{0}\right\rangle_{2, \Gamma}=\langle\mu(t), \bar{\phi}(t)\rangle_{2}-\alpha\left\langle\bar{\phi}(t), \partial_{t} \phi(t)\right\rangle_{2} \\
-\left\langle\bar{\psi}(t), \partial_{t} \psi(t)\right\rangle_{2, \Gamma}+\delta\langle\bar{\phi}(t), \theta(t)\rangle_{2} .
\end{gathered}
$$

The first term on the right-hand side of (3.22) can be estimated by $1 / 2\|\nabla \phi(t)\|_{2}^{2}+$ $C\|\nabla \mu\|_{2}^{2}$, since $\langle\bar{\phi}(t)\rangle=0$. Consequently, the estimates (3.18), (3.21) and (3.22) yield

$$
\begin{gathered}
\|\nabla \phi(t)\|_{2}^{2}+\left\|\nabla_{\Gamma} \psi(t)\right\|_{2, \Gamma}^{2}+\left\langle F_{1}^{\prime}(\phi(t)), \bar{\phi}(t)\right\rangle_{2}+\left\langle F_{2}^{\prime}(\psi(t)), \bar{\psi}(t)\right\rangle_{2, \Gamma} \\
\leq Q_{*}\left(\|(\phi(0), \psi(0), \theta(0))\|_{\mathbb{Y}_{K}^{\alpha}}^{2}\right) e^{-\rho t}+C_{4},
\end{gathered}
$$

where $Q_{*}$ and $C_{4}$ are independent of $t$. It remains to observe that due to the assumption (3.10) on the nonlinearities $F_{i}, i=1,2$, we obtain

$$
\begin{aligned}
& \|\psi(t)\|_{1, \Gamma}+\left\|F_{1}^{\prime}(\phi(t))\right\|_{1}+\left\|F_{2}^{\prime}(\psi(t))\right\|_{1, \Gamma} \\
& \leq Q_{*}\left(\|(\phi(0), \psi(0), \theta(0))\|_{\mathbb{Y}_{K}^{\alpha}}^{2}\right) e^{-\rho t}+C_{5} .
\end{aligned}
$$

Thus, from (3.21) and (3.24), we have the required estimate for the $H^{1}$-norm of $\mu$, that is,

$$
\langle\mu(t)\rangle^{2} \leq Q_{3}\left(\|(\phi(0), \psi(0), \theta(0))\|_{\mathbb{Y}_{K}^{\alpha}}^{2}\right) e^{-\rho t}+C_{6} .
$$

Collecting now (3.21) and (3.25), we have verified the $\mu$-part of the estimate in (3.20). It remains to obtain the required estimates of $\phi, \psi$ and $\theta$ in the $H^{2}$-norms. To do so, we can rewrite (for every fixed $t$ ) our problem $(2.5),(2.8)$ as a second order 
nonlinear elliptic problem where the chemical potential and the dynamic terms are considered as external forces. We have

$$
\left\{\begin{array}{c}
-\Delta \phi(t)+F_{1}^{\prime}(\phi(t))=g_{1}(t):=\mu(t)-\alpha \partial_{t} \phi(t)+\delta \theta(t), \phi_{\mid \Gamma}=\psi \\
-\Delta_{\Gamma} \psi(t)+\psi+\partial_{n} \phi(t)+F_{2}^{\prime}(\psi(t))=g_{2}(t):=-\partial_{t} \psi(t) .
\end{array}\right.
$$

We notice that the estimates (3.12), (3.21), and (3.25) imply that

$$
\left\|g_{1}(t)\right\|_{2}^{2}+\left\|g_{2}(t)\right\|_{2, \Gamma}^{2} \leq Q_{3}\left(\|(\phi(0), \psi(0), \theta(0))\|_{\mathbb{Y}_{K}^{\alpha}}^{2}\right) e^{-\rho t}+C_{6} .
$$

Applying now the maximum principle (see, Lemma A.2, $[\mathbf{4 1}]$ ) to problem (3.26), we obtain

$$
\begin{gathered}
\|\phi(t)\|_{\infty}^{2}+\|\psi(t)\|_{\infty, \Gamma}^{2} \leq C_{F_{i}}+\left\|g_{1}(t)\right\|_{2}^{2}+\left\|g_{2}(t)\right\|_{2, \Gamma}^{2} \\
\leq Q_{3}\left(\|(\phi(0), \psi(0), \theta(0))\|_{\mathbb{Y}_{K}^{\alpha}}^{2}\right) e^{-\rho t}+C_{7},
\end{gathered}
$$

where $C_{7}>0$ is independent of $t$. Finally, applying the above estimate combined with a $H^{2}$ - regularity theorem (Lemma A.1, $[\mathbf{4 1}]$ ) to the elliptic boundary value problem (3.26), but with the nonlinearities $F_{i}^{\prime}$ acting as external forces, we easily deduce that

$$
\|\phi(t)\|_{H^{2}}^{2}+\|\psi(t)\|_{H^{2}(\Gamma)}^{2} \leq Q_{4}\left(\|(\phi(0), \psi(0), \theta(0))\|_{\mathbb{Y}_{K}^{\alpha}}^{2}\right) e^{-\rho t}+C_{8},
$$

where $Q_{4}$ is a monotonic function independent of $t$.

In order to obtain the $H^{2}$ - estimate for the temperature function $\theta$, we apply the standard parabolic maximum principle to the parabolic equation (cf. [40], $[42])$ :

$$
\partial_{t} \theta-\Delta \theta+F_{3}^{\prime}(\theta)=h_{3}(t):=-\delta \partial_{t} \phi(t), \theta_{\mid t=0}=\theta_{0},
$$

when $\theta$ satisfies Dirichlet, Neumann and Robin boundary conditions. Moreover, it follows from (3.12) that

$$
\left\|h_{3}(t)\right\|_{2}^{2} \leq Q\left(\|(\phi(0), \psi(0), \theta(0))\|_{\mathbb{Y}_{K}^{\alpha}}^{2}\right) e^{-\eta t}+C,
$$

where $Q$ and $C$ are independent of $t$. Since, we also know that $A_{K}=-\Delta$, when $K \in\{D, N, R\}$, generates a bounded analytic semigroup on $H^{2}(\Omega) \subset L^{\infty}(\Omega)$ (since $n=3$ ) and $F_{3}^{\prime}(\theta) \theta \geq-\zeta_{1}\left(\zeta_{1}>0\right)$, if $K \in\{D, R\}$, while $F_{3}^{\prime}(\theta) \theta \geq \zeta_{2} \theta^{2}-\zeta_{3}$, $\left(\zeta_{2}>0, \zeta_{3} \geq 0\right)$, when $K=N$ (also note, from (1.12), that $F_{3}^{\prime}(s)$ is monotonic, if $|s|$ is large enough), it follows that

$$
\|\theta(t)\|_{\infty} \leq \bar{C}\|\theta(0)\|_{H^{2}} e^{-\eta t}+\bar{C} \sup _{s \in[0, t]} e^{-\eta(t-s)}\left\|h_{3}(t)\right\|_{2},
$$

for suitable constants $\bar{C}$ and $\eta$ (see [40, Chapter 1, Section 2] for details; cf. [42] also). Inserting now the $L^{2}$-estimate for $h_{3}(t)$ from (3.30) into (3.31), we obtain

$$
\|\theta(t)\|_{\infty} \leq Q_{\alpha}\left(\|(\phi(0), \psi(0), \theta(0))\|_{\mathbb{Y}_{K}^{\alpha}}^{2}\right) e^{-\rho t}+C_{\alpha},
$$

where the new function $Q_{\alpha}$ and $C_{\alpha}, \rho$ are independent of $t$. Applying now the standard parabolic regularity theorem (see e.g., $[\mathbf{4 2},(1.23)-(1.37)])$ to

$$
\partial_{t} \theta-\Delta \theta=h_{4}(t):=-\delta \partial_{t} \phi-F_{3}^{\prime}(\theta), \theta_{\mid t=0}=\theta_{0},
$$


and recalling the above estimates $(3.31),(3.32)$ and the embedding $H^{2}(\Omega) \subset$ $L^{\infty}(\Omega)$, we obtain

$$
\|\theta(t)\|_{H^{2}}+\left\|\partial_{t} \theta(t)\right\|_{2} \leq Q_{\alpha}\left(\|(\phi(0), \psi(0), \theta(0))\|_{\mathbb{Y}_{K}^{\alpha}}^{2}\right) e^{-\rho_{2} t}+C_{\alpha},
$$

where the new monotonic function $Q_{\alpha}$ and the constants $\rho_{2}, C_{\alpha}$ are independent of $t$. Nevertheless, the function $C_{\alpha}$ depends on $M_{0}$. Thus, we have obtained the required uniform estimate for the solution in $\mathbb{Y}_{K}^{\alpha}$. This finishes the proof of the theorem.

Let us now denote $\mathbb{Z}_{q}:=H^{q}(\Omega) \times H^{q}(\Gamma) \times H^{q}(\Omega), q \geq 1$, endowed with the natural norms in $H^{q}$. In the sequel, we will also derive uniform bounds on the solutions $(\phi, \psi, \theta)$ in $\mathbb{Z}_{3}$ which are given in the following theorem.

TheOREM 5. Let the assumptions of Theorem 4 hold and let $(\phi(t), \psi(t), \theta(t))$ be a solution of $(2.4)-(2.10)$. Then

$$
(\phi(t), \psi(t), \theta(t)) \in \mathbb{Z}_{3},
$$

for every $t>0$ and the following estimate holds:

$$
\|\phi(t)\|_{H^{3}}^{2}+\|\psi(t)\|_{H^{3}(\Gamma)}^{2}+\|\theta(t)\|_{H^{3}}^{2} \leq \frac{t+1}{t^{2}} Q_{\alpha}\left(\|(\phi(0), \psi(0), \theta(0))\|_{\mathbb{Y}_{K}^{\alpha}}^{2}\right),
$$

for every $t \in\left[t_{0}, 1\right], t_{0}>0$. Here the monotonic function $Q_{\alpha}$ is independent of $t$, but depends on $\alpha$.

Proof. As in the proof of Proposition 3, we differentiate $(2.4)-(2.9)$ and define

$$
(u(t), v(t), \varpi(t), m(t)):=\left(\partial_{t} \phi(t), \partial_{t} \psi(t), \partial_{t} \theta(t), \partial_{t} \mu(t)\right) .
$$

These functions satisfy (3.13) and

$$
\left\{\begin{array}{c}
\partial_{t} \varpi(t)-\Delta \varpi(t)=-\delta \partial_{t} u(t)-F_{3}^{\prime \prime}(\theta(t)) \varpi(t) \text { in } \Omega, \\
b \partial_{n} \varpi(t)+c \varpi(t)=0 \text { on } \Gamma .
\end{array}\right.
$$

Now, multiplying the first, third and fourth equations of (3.13) scalarly by

$$
t A_{N}^{-1}\left(\partial_{t} u(t)\right), t \partial_{t} u(t) \text { and } t \partial_{t} v(t)
$$

respectively and the first equation of (3.35) by $t \varpi(t)$, then integrating by parts and using the boundary condition of (3.35), we obtain after standard transformations:

$$
\begin{gathered}
t\left(\left\langle A_{K} \varpi(t), \varpi(t)\right\rangle_{2}+\alpha\left\|\partial_{t} u(t)\right\|_{2}^{2}+\left\|\partial_{t} u(t)\right\|_{H^{-1}}^{2}+\left\|\partial_{t} v(t)\right\|_{2, \Gamma}^{2}\right) \\
+\frac{1}{2} \frac{d}{d t}\left[t\left(\|u(t)\|_{H^{1}}^{2}+\|v(t)\|_{H^{1}(\Gamma)}^{2}+\|\varpi(t)\|_{2}^{2}\right)\right] \\
=\frac{1}{2}\left(\|u(t)\|_{H^{1}}^{2}+\|v(t)\|_{H^{1}(\Gamma)}^{2}+\|\varpi(t)\|_{2}^{2}\right)-\left\langle F_{1}^{\prime \prime}(\phi(t)) u(t), t \partial_{t} u(t)\right\rangle_{2} \\
-\left\langle F_{2}^{\prime \prime}(\psi(t)) v(t), t \partial_{t} v(t)\right\rangle_{2, \Gamma}-\left\langle F_{3}^{\prime \prime}(\theta(t)) \varpi(t), t \varpi(t)\right\rangle_{2} .
\end{gathered}
$$

We estimate the last three terms on the right-hand side of (3.36) as follows:

$$
\begin{gathered}
\left|\left\langle F_{1}^{\prime \prime}(\phi(t)) u(t), t \partial_{t} u(t)\right\rangle_{2}\right| \leq C t\left\|F_{1}^{\prime \prime}(\phi(t)) u(t)\right\|_{H^{1}}\left\|\partial_{t} u(t)\right\|_{H^{-1}} \\
\leq \frac{t}{2}\left\|\partial_{t} u(t)\right\|_{H^{-1}}^{2}+Q_{9}\left(\|\phi(t)\|_{H^{2}}\right)\left\|\partial_{t} \phi(t)\right\|_{H^{1}}^{2} .
\end{gathered}
$$


The estimate for the last term is much simpler since

$$
\begin{gathered}
\left|\left\langle F_{2}^{\prime \prime}(\psi(t)) v(t), t \partial_{t} v(t)\right\rangle_{2, \Gamma}\right| \leq t\left\|F_{2}^{\prime \prime}(\psi(t)) v(t)\right\|_{2, \Gamma}\left\|\partial_{t} v(t)\right\|_{2, \Gamma} \\
\leq \frac{t}{2}\left\|\partial_{t} v(t)\right\|_{2, \Gamma}^{2}+Q_{9}\left(\|\psi(t)\|_{H^{2}(\Gamma)}\right)\left\|\partial_{t} \psi(t)\right\|_{2, \Gamma}^{2} .
\end{gathered}
$$

The last term on the right-hand side of (3.36) can be estimated analogously. Note, however, that (1.12) implies that $F_{3}^{\prime \prime}(s) \geq-C$, thus, for $t \in[0,1]$, we have

$$
-\left\langle F_{3}^{\prime \prime}(\theta(t)) \varpi(t), t \varpi(t)\right\rangle_{2} \leq C\left\|\partial_{t} \theta(t)\right\|_{2}^{2} .
$$

Integrating now (3.36) in $t$ and recalling the above estimates and collecting (3.33), $(3.12),(3.28)$ and $(3.20)$, we obtain

$$
\begin{gathered}
t\left(\left\|\partial_{t} \phi(t)\right\|_{H^{1}}^{2}+\left\|\partial_{t} \psi(t)\right\|_{H^{1}(\Gamma)}^{2}+\left\|\partial_{t} \theta(t)\right\|_{2}^{2}\right)+\int_{0}^{t} s\left\langle A_{K} \partial_{t} \theta(s), \partial_{t} \theta(s)\right\rangle_{2} d s \\
+\int_{0}^{t} s\left(\alpha\left\|\partial_{t}^{2} \phi(t)\right\|_{2}^{2}+\left\|\partial_{t}^{2} \phi(t)\right\|_{H^{-1}}^{2}+\left\|\partial_{t}^{2} \psi(t)\right\|_{2, \Gamma}^{2}\right) d s \\
\leq Q_{10}\left(\|(\phi(0), \psi(0), \theta(0))\|_{\mathbb{Y}_{K}^{\alpha}}^{2}\right),
\end{gathered}
$$

$t \in[0,1]$, for some monotonic function $Q_{10}$ which is independent of $t$. Having obtained estimate (3.40), we can rewrite the equations $(2.5),(2.8)$ as a linear elliptic boundary value problem:

$$
\left\{\begin{array}{c}
-\Delta \phi(t)=g_{3}(t):=\mu(t)-\alpha \partial_{t} \phi(t)+\delta \theta(t)-F_{1}^{\prime}(\phi(t)), \phi(t)_{\mid \Gamma}=\psi(t) \\
-\Delta_{\Gamma} \psi(t)+\psi+\partial_{n} \phi(t)=g_{4}(t):=-\partial_{t} \psi(t)-F_{2}^{\prime}(\psi(t)) .
\end{array}\right.
$$

According to the $H^{3}$ regularity theorem (see [41]) for (3.41), we have

$$
\|\phi(t)\|_{H^{3}}^{2}+\|\psi(t)\|_{H^{3}(\Gamma)}^{2} \leq C\left(\left\|g_{3}(t)\right\|_{H^{1}}^{2}+\left\|g_{4}(t)\right\|_{H^{1}(\Gamma)}^{2}\right),
$$

where $C>0$ is independent of the solution $(\phi, \psi)$ and $t$. Moreover, according to the estimates $(3.12),(3.21),(3.25),(3.27)-(3.28)$ and $(3.40)$, we have

$$
\left\|g_{3}(t)\right\|_{H^{1}}^{2}+\left\|g_{4}(t)\right\|_{H^{1}(\Gamma)}^{2} \leq \frac{1}{t} Q_{11}\left(\|(\phi(0), \psi(0), \theta(0))\|_{\mathbb{Y}_{K}^{\alpha}}^{2}\right), t \in(0,1]
$$

for some monotonic function $Q_{11}$. Combining now estimate (3.42) with (3.43), we obtain the required $H^{3}$ - estimate for the function $(\phi, \psi)$ in $(3.34)$.

Next, we multiply the first equation of (3.35) scalarly by $t^{2} \partial_{t} \varpi(t)$, then integrate over $\Omega$, and use the boundary condition of (3.35) once more. After standard transformations, we deduce that

$$
\begin{gathered}
t^{2}\left\|\partial_{t} \varpi(t)\right\|_{2}^{2}+\frac{1}{2} \frac{d}{d t}\left[t^{2}\left\langle A_{K} \varpi(t), \varpi(t)\right\rangle_{2}\right]=-\delta\left\langle\partial_{t} u(t), t^{2} \partial_{t} \varpi(t)\right\rangle_{2} \\
-\left\langle F_{3}^{\prime \prime}(\theta(t)) \varpi(t), t^{2} \partial_{t} \varpi(t)\right\rangle_{2}+t\left\langle A_{K} \varpi(t), \varpi(t)\right\rangle_{2} .
\end{gathered}
$$

We estimate the first two terms on the right-hand side of (3.44) as follows:

$$
\delta\left|\left\langle\partial_{t} u(t), t^{2} \partial_{t} \varpi(t)\right\rangle_{2}\right| \leq \frac{t^{2}}{4}\left\|\partial_{t} \varpi(t)\right\|_{2}^{2}+C t^{2}\left\|\partial_{t} u(t)\right\|_{2}^{2},
$$




$$
\left|\left\langle F_{3}^{\prime \prime}(\theta(t)) \varpi(t), t^{2} \partial_{t} \varpi(t)\right\rangle_{2}\right| \leq \frac{t^{2}}{4}\left\|\partial_{t} \varpi(t)\right\|_{2}^{2}+t^{2} Q\left(\|\theta(t)\|_{\infty}\right)\|\varpi(t)\|_{2}^{2} .
$$

Inserting now (3.45) and (3.46) in (3.44), then integrating in $t \in[0,1]$ the relation that we obtain, we deduce from (3.44) that

$$
t^{2}\left\langle A_{K} \partial_{t} \theta(t), \partial_{t} \theta(t)\right\rangle_{2}+\int_{0}^{t} s^{2}\left\|\partial_{t}^{2} \theta(s)\right\|_{2}^{2} d s \leq C \int_{0}^{t} s\left\|\partial_{t}^{2} \phi(s)\right\|_{2}^{2} d s+
$$

$$
+Q_{\alpha}\left(\|(\phi(0), \psi(0), \theta(0))\|_{\mathbb{Y}_{K}^{\alpha}}^{2}\right) \int_{0}^{t} s^{2}\left\|\partial_{t} \theta(s)\right\|_{2}^{2} d s+\int_{0}^{t} s\left\langle A_{K} \partial_{t} \theta(t), \partial_{t} \theta(t)\right\rangle_{2} d s,
$$

where $Q_{\alpha}$ is a monotonic function that is independent $t$, but depends on $\alpha$. Using now (3.40) to estimate the last term on the right-hand side of (3.47), collecting the estimate for $\left\|\partial_{t} \theta(t)\right\|_{2}^{2}$ from (3.40), we obtain

$$
\left\|\partial_{t} \theta(t)\right\|_{Z_{K}}^{2} \leq \frac{t+1}{t^{2}} Q_{\alpha}\left(\|(\phi(0), \psi(0), \theta(0))\|_{\mathbb{Y}_{K}^{\alpha}}^{2}\right), t \in(0,1],
$$

where $Q_{\alpha}$ is a new function that depends on $\alpha$, but it is independent of $t$. Analogously, we can rewrite $(2.6),(2.9)$ as a linear elliptic boundary value problem:

$$
\left\{\begin{array}{c}
-\Delta \theta(t)=g_{5}(t) \\
b \partial_{n} \theta(t)+c \theta(t)=0
\end{array}\right.
$$

where $g_{5}(t):=-\partial_{t} \theta(t)-\delta \partial_{t} \phi(t)-F_{3}^{\prime}(\theta(t))$. Standard elliptic theory for problem (3.49) implies that

$$
\|\theta(t)\|_{H^{3}}^{2} \leq C\left(\left\|g_{5}(t)\right\|_{H^{1}}^{2}+\|\theta(t)\|_{H^{2}}^{2}\right)
$$

which together with the estimates $(3.40),(3.48)$ yields the required estimate on the $H^{3}$ - norm of $\theta$, that is, we obtain

$$
\|\theta(t)\|_{H^{3}}^{2} \leq \frac{t+1}{t^{2}} Q_{\alpha}\left(\|(\phi(0), \psi(0), \theta(0))\|_{\mathbb{Y}_{K}^{\alpha}}^{2}\right), t \in(0,1] .
$$

This finishes the proof of Theorem 5 .

In the sequel, we will also verify the uniqueness of the solution and the Lipschitz continuity with respect to the initial data.

Theorem 6. Let $K \in\{D, N, R\}$. Let the assumptions of Theorem 4 hold and let the functions $\left(\phi_{1}(t), \psi_{1}(t), \theta_{1}(t)\right)$ and $\left(\phi_{2}(t), \psi_{2}(t), \theta_{2}(t)\right)$ be two solutions of problem $(2.4)-(2.10)$. Set

$$
(\bar{\phi}, \bar{\psi}, \bar{\theta}):=\left(\phi_{1}-\phi_{2}, \psi_{1}-\psi_{2}, \theta_{1}-\theta_{2}\right) .
$$

Then the following estimate holds:

$$
\begin{gathered}
\left\|\phi_{1}(t)-\phi_{2}(t)\right\|_{H^{1}}^{2}+\left\|\psi_{1}(t)-\psi_{2}(t)\right\|_{H^{1}(\Gamma)}^{2}+\left\|\theta_{1}(t)-\theta_{2}(t)\right\|_{2}^{2} \\
+\int_{0}^{t}\left(\alpha\left\|\partial_{t} \bar{\phi}(s)\right\|_{2}^{2}+\left\|\partial_{t} \bar{\phi}(s)\right\|_{H^{-1}}^{2}+\left\langle A_{K} \bar{\theta}(s), \bar{\theta}(s)\right\rangle_{2}+\left\|\partial_{t} \bar{\psi}(s)\right\|_{2, \Gamma}^{2}\right) d s \\
\leq C e^{L t}\left(\left\|\phi_{1}(0)-\phi_{2}(0)\right\|_{H^{1}}^{2}+\left\|\psi_{1}(0)-\psi_{2}(0)\right\|_{H^{1}(\Gamma)}^{2}+\left\|\theta_{1}(0)-\theta_{2}(0)\right\|_{2}^{2}\right),
\end{gathered}
$$


where the positive constants $C$ and $L$ are independent of $t$, but depend on the initial data and $\alpha$.

Proof. First, set $\mu:=\mu_{1}-\mu_{2}$. The functions $\bar{\phi}(t), \bar{\psi}(t), \bar{\theta}(t), \mu(t)$ satisfy

$$
\begin{gathered}
\left\{\begin{array}{c}
\partial_{t} \bar{\phi}(t)=\Delta \mu(t) \text { in } \Omega \times(0, \infty), \partial_{n} \mu(t)=0 \text { on } \Gamma \\
\mu(t)=\alpha \partial_{t} \bar{\phi}(t)-\Delta \bar{\phi}(t)+l_{1}(t) \bar{\phi}(t)-\delta \bar{\theta}(t) \text { in } \Omega \\
\partial_{t} \bar{\psi}(t)=\Delta_{\Gamma} \bar{\psi}(t)-\partial_{n} \bar{\phi}(t)-\bar{\psi}(t)-l_{2}(t) \bar{\psi}(t) \text { on } \Gamma, \bar{\phi}(t)_{\mid \Gamma}=\bar{\psi}(t)
\end{array}\right. \\
\left\{\begin{array}{c}
\partial_{t} \bar{\theta}(t)-\Delta \bar{\theta}(t)=-\delta \partial_{t} \bar{\phi}(t)-l_{3}(t) \bar{\theta}(t) \text { in } \Omega \\
b \partial_{n} \bar{\theta}(t)+c \bar{\theta}(t)=0 \text { on } \Gamma,
\end{array}\right. \\
\bar{\phi}_{\mid t=0}=\phi_{1}(0)-\phi_{2}(0), \bar{\psi}_{\mid t=0}=\psi_{1}(0)-\psi_{2}(0), \bar{\theta}_{\mid t=0}=\theta_{1}(0)-\theta_{2}(0),
\end{gathered}
$$

where

$$
l_{j}(t):=\int_{0}^{1} F_{j}^{\prime \prime}\left(s v_{1}(t)+(1-s) v_{2}(t)\right) d s, \quad j=1,2,3 .
$$

The definition of the functions $v_{i}$ is as follows: when $j=1, v_{i}=\phi_{i}$, for $i=1$, 2 , when $j=2, v_{i}=\psi_{i}$, for $i=1,2$, whereas, when $j=3, v_{i}=\theta_{i}, i=1,2$. It now follows from the estimates of Propositions 2, 3, Theorem 4 and from the embeddings $H^{2}(\Omega) \subset L^{\infty}(\Omega), H^{2}(\Gamma) \subset L^{\infty}(\Gamma)$ that

$$
\left\|\partial_{t} l_{1}(t)\right\|_{2}+\left\|\partial_{t} l_{2}(t)\right\|_{2, \Gamma}+\left\|l_{1}(t)\right\|_{H^{2}}+\left\|l_{2}(t)\right\|_{H^{2}(\Gamma)}+\left\|\partial_{t} l_{3}(t)\right\|_{2}
$$

$+\left\|l_{3}(t)\right\|_{H^{2}} \leq L:=Q_{\alpha}\left(\left\|\left(\phi_{1}(0), \psi_{1}(0), \theta_{1}(0)\right)\right\|_{\mathbb{Y}_{K}^{\alpha}}+\left\|\left(\phi_{2}(0), \psi_{2}(0), \theta_{2}(0)\right)\right\|_{\mathbb{Y}_{K}^{\alpha}}\right)$,

for a monotonic function $Q_{\alpha}$ that is independent of $t$, but depends on $\alpha$. Also, note that $\left\langle\partial_{t} \bar{\phi}(t)\right\rangle=0$, due to (2.1). Multiplying now the first, third and fourth equations of (3.53) scalarly by $A_{N}^{-1}\left(\partial_{t} \bar{\phi}(t)\right), \partial_{t} \bar{\phi}(t)$ and $\partial_{t} \bar{\psi}(t)$, respectively, then the first equation of (3.54) scalarly by $\bar{\theta}(t)$, integrating by parts, and using the boundary condition of (3.54), we obtain after standard transformations:

$$
\begin{gathered}
\frac{1}{2} \frac{d}{d t}\left[\|\bar{\phi}(t)\|_{H^{1}}^{2}+\|\bar{\psi}(t)\|_{H^{1}(\Gamma)}^{2}+\|\bar{\theta}(t)\|_{2}^{2}\right]+\alpha\left\|\partial_{t} \bar{\phi}(t)\right\|_{2}^{2}+\left\|\partial_{t} \bar{\phi}(t)\right\|_{H^{-1}}^{2} \\
+\left\langle A_{K} \bar{\theta}(t), \bar{\theta}(t)\right\rangle_{2}+\left\|\partial_{t} \bar{\psi}(t)\right\|_{2, \Gamma}^{2}=-\left\langle l_{1}(t) \bar{\phi}(t), \partial_{t} \bar{\phi}(t)\right\rangle_{2}-\left\langle l_{2}(t) \bar{\psi}(t), \partial_{t} \bar{\psi}(t)\right\rangle_{2, \Gamma} \\
(3.56) \quad-\left\langle l_{3}(t) \bar{\theta}(t), \bar{\theta}(t)\right\rangle_{2}:=\mathcal{F}(\bar{\phi}, \bar{\psi}, \bar{\theta}) .
\end{gathered}
$$

It now follows from the assumptions $(1.12)-(1.14)$ on the nonlinearities $F_{i}(i=$ $1,2,3)$ and the embeddings $H^{2}(\Omega) \subset L^{\infty}(\Omega), H^{2}(\Gamma) \subset L^{\infty}(\Gamma)$ that

$$
\begin{gathered}
|\mathcal{F}(\bar{\phi}, \bar{\psi}, \bar{\theta})| \leq \frac{1}{\alpha}\left[Q\left(\left\|\phi_{i}(t)\right\|_{H^{2}}\right)\|\bar{\phi}(t)\|_{2}^{2}+Q\left(\left\|\psi_{i}(t)\right\|_{H^{2}(\Gamma)}\right)\|\bar{\psi}(t)\|_{2, \Gamma}^{2}\right] \\
+\frac{\alpha}{2}\left\|\partial_{t} \bar{\phi}(t)\right\|_{2}^{2}+\frac{1}{2}\left\|\partial_{t} \bar{\psi}(t)\right\|_{2, \Gamma}^{2}+Q\left(\left\|\theta_{i}(t)\right\|_{H^{2}}\right)\|\bar{\theta}(t)\|_{2}^{2},
\end{gathered}
$$

where the monotonic function $Q$ is independent of $t, \alpha$ and $i=1,2$. Moreover, inserting the above estimate into the right-hand side of (3.56) and using the estimates (3.12), (3.20), (3.55), we obtain

$$
\frac{d}{d t}\left[\|\bar{\phi}(t)\|_{H^{1}}^{2}+\|\bar{\psi}(t)\|_{H^{1}(\Gamma)}^{2}+\|\bar{\theta}(t)\|_{2}^{2}\right]+2 \alpha\left\|\partial_{t} \bar{\phi}(t)\right\|_{2}^{2}+2\left\|\partial_{t} \bar{\phi}(t)\right\|_{H^{-1}}^{2}
$$




$$
+2\left\langle A_{K} \bar{\theta}(t), \bar{\theta}(t)\right\rangle_{2}+2\left\|\partial_{t} \bar{\psi}(t)\right\|_{2, \Gamma}^{2} \leq L\left[\|\bar{\phi}(t)\|_{H^{1}}^{2}+\|\bar{\psi}(t)\|_{H^{1}(\Gamma)}^{2}+\|\bar{\theta}(t)\|_{2}^{2}\right] .
$$

Applying a suitable version of Gronwall's inequality to relation (3.57), we easily obtain our conclusion (3.52). The proof is finished.

We close this section with the proof of the existence of solutions to our problem (2.4)-(2.10), which was conveniently rewritten as (3.1)-(3.3) in the beginning of this section. This can be proved based on the a priori estimates derived in this section and by a Faedo-Galerkin approximation scheme. To this end, let us consider the operator $\mathcal{B}_{0}: D\left(\mathcal{B}_{0}\right) \subset \mathbb{V}_{0} \rightarrow \mathbb{V}_{0}$ given formally by

$$
\mathcal{B}_{0}\left(\begin{array}{l}
\phi \\
\psi
\end{array}\right)=\left(\begin{array}{c}
-\Delta \phi \\
-\Delta_{\Gamma} \psi+\partial_{n} \phi+\psi
\end{array}\right)
$$

with domain $D\left(\mathcal{B}_{0}\right)=\left\{\Theta=(\phi, \psi): \phi \in C^{2}(\bar{\Omega}), \psi=\left.\phi\right|_{\Gamma}\right\}$. It is an easy exercise to show that we have $\left\langle\mathcal{B}_{0} \Theta, \Xi\right\rangle_{\mathbb{V}_{0}}=\langle\Theta, \Xi\rangle_{\mathbb{V}_{1}}$, for all $\Theta \in D\left(\mathcal{B}_{0}\right)$ and all $\Xi \in \mathbb{V}_{1}$. It follows from a well-known result (see, e.g., [25], cf. also [48]) that the closed bilinear form $\varrho(\Theta, \Xi):=\langle\Theta, \Xi\rangle_{\mathbb{V}_{1}}$ defines a strictly positive self-adjoint unbounded operator $\mathcal{B}: D(\mathcal{B})=\left\{\Theta \in \mathbb{V}_{1}: \mathcal{B} \Theta \in \mathbb{V}_{0}\right\} \rightarrow \mathbb{V}_{0}$, such that, for any $\Xi \in \mathbb{V}_{1}$, we have $\langle\mathcal{B} \Theta, \Xi\rangle_{\mathbb{V}_{0}}=\varrho(\Theta, \Xi)$. Thus, for $i \in \mathbf{N}$, we take a complete system of eigenfunctions $\left\{\Theta_{i}=\left(\phi_{i}, \psi_{i}\right)\right\}_{i}$ of the problem $\mathcal{B} \Theta_{i}=\widehat{\lambda}_{i} \Theta_{i}$ in $\mathbb{V}_{0}$ with $\Theta_{i} \in D(\mathcal{B})$. Moreover, recall that $A_{K}=-\Delta$, when $K \in\{D, N, R\}$ is nonnegative and selfadjoint on $L^{2}(\Omega)$. Then, we have (for $i \in \mathbf{N}$ ) a complete system of eigenfunctions $\left\{\theta_{i}^{K}\right\}_{i}$ of the problem $A_{K} \theta_{i}=\lambda_{i}^{K} \theta_{i}^{K}$ in $L^{2}(\Omega)$ with $\theta_{i}^{K} \in D\left(A_{K}\right)$. According to the general spectral theory, the eigenvalues $\widehat{\lambda}_{i}$ and $\lambda_{i}^{K}, K \in\{D, N, R\}$ can be increasingly ordered and counted according to their multiplicities in order to form a real divergent sequence. Moreover, the respective eigenvectors $\Theta_{i}$ and $\theta_{i}^{K}$ turn out to form an orthogonal basis in $\mathbb{V}_{1}, \mathbb{V}_{0}$ and $L^{2}(\Omega), H^{1}(\Omega)$ respectively. The eigenvectors $\Theta_{i}$ and $\theta_{i}^{K}$ may be assumed to be normalized in the norm of $\mathbb{V}_{0}$ and $L^{2}(\Omega)$ respectively. At this point, we set the spaces

$$
\begin{gathered}
\mathcal{K}_{n}=\operatorname{span}\left\{\Theta_{1}, \Theta_{2}, \ldots, \Theta_{n}\right\}, \mathcal{K}_{\infty}=\cup_{n=1}^{\infty} \mathcal{K}_{n}, \\
\mathcal{P}_{n}^{K}=\operatorname{span}\left\{\theta_{1}^{K}, \theta_{2}^{K}, \ldots, \theta_{n}^{K}\right\}, \mathcal{P}_{\infty}^{K}=\cup_{n=1}^{\infty} \mathcal{P}_{n}^{K} .
\end{gathered}
$$

Clearly, $\mathcal{K}_{\infty}$ and $\mathcal{P}_{\infty}^{K}, K \in\{D, N, R$,$\} are dense subspaces of \mathbb{V}_{1}, \mathbb{V}_{2}$ and $H^{1}(\Omega)$, $D\left(A_{K}\right)$ respectively. For any $n \in \mathbf{N}$, we look for functions of the form

$$
\Theta=\Theta_{n}=\sum_{i=1}^{n} d_{i}(t) \Theta_{i}, \theta^{K}=\theta_{n}^{K}=\sum_{i=1}^{n} e_{i}(t) \theta_{i}^{K}
$$

solving the approximate problem that we will introduce below. Note that the chemical potential $\mu$ can be found in terms of $\Theta_{n}$ and $\theta_{n}^{K}$ from the boundary value problem $(2.2)-(2.3)$. That is, it is enough to solve for $\Theta_{n}$ and $\theta_{n}^{K}$. Furthermore, we also define the function

$$
H(t):=-\frac{|\Gamma|}{|\Omega|} \sum_{i=1}^{n} d_{i}(t)\left\langle\partial_{n} \phi_{i}\right\rangle_{\Gamma}+\left\langle F_{1}^{\prime}\left(\sum_{i=1}^{n} d_{i}(t) \phi_{i}\right)\right\rangle-\delta \sum_{i=1}^{n} e_{i}(t)\left\langle\theta_{i}^{K}\right\rangle,
$$

to account for the contribution of the last term on the right-hand side of first equation of (3.1). Note that from (3.58), we have

$$
\phi=\phi_{n}=\sum_{i=1}^{n} d_{i}(t) \phi_{i}, \psi=\psi_{n}=\sum_{i=1}^{n} d_{i}(t) \psi_{i} .
$$


Also, in the definition of $\Theta_{n}$ and $\theta_{n}^{K}, d_{i}(t)$ and $e_{i}(t)$ are sought to be suitably regular real valued functions. As approximations for the initial data $\Phi_{0}=\left(\phi_{0}, \psi_{0}, \theta_{0}\right)$, we take

$$
\Phi_{n 0}=\left(\phi_{n 0}, \psi_{n 0}, \theta_{n 0}\right) \in \mathbb{Y}_{\alpha}^{K}, \text { such that } \lim _{n \rightarrow \infty} \Phi_{n 0}=\Phi_{0} \text { in } \mathbb{Y}_{\alpha}^{K} .
$$

Before we introduce our problem, let us define the following operators $\mathcal{F}: D(\mathcal{F}) \subset$ $\mathbb{V}_{0} \rightarrow \mathbb{V}_{0}, \widehat{\mathcal{F}}: D(\widehat{\mathcal{F}}) \subset L^{2}(\Omega) \rightarrow L^{2}(\Omega), \mathcal{G}: D(\mathcal{G}) \subset \mathbb{V}_{0} \rightarrow \mathbb{V}_{0}$ and $\mathcal{N}: \mathbb{V}_{0} \rightarrow \mathbb{V}_{0}$ such that

$$
\begin{gathered}
\mathcal{F}\left(\begin{array}{l}
\phi \\
\psi
\end{array}\right)=\left(\begin{array}{l}
F_{1}^{\prime}(\phi) \\
F_{2}^{\prime}(\psi)
\end{array}\right), \widehat{\mathcal{F}}(\theta)=F_{3}^{\prime}(\theta), \mathcal{G}\left(\begin{array}{l}
\phi \\
\psi
\end{array}\right)=\left(\begin{array}{c}
H \\
0
\end{array}\right), \\
\mathcal{N}\left(\begin{array}{l}
\phi \\
\psi
\end{array}\right)=\left(\begin{array}{c}
\left(\alpha+A_{N}^{-1}\right) \phi \\
\psi
\end{array}\right) .
\end{gathered}
$$

The problem that we must solve is given by $\left(\mathbb{P}_{n}^{K}\right)$, for a fixed $K \in\{D, N, R$,$\} and$ for any $n \geq 1$,

$$
\left\{\begin{array}{c}
\left\langle\partial_{t} \mathcal{N}\left(\Theta_{n}\right), \bar{\Theta}\right\rangle_{\mathbb{V}_{0}}+\left\langle\mathcal{B} \Theta_{n}, \bar{\Theta}\right\rangle_{\mathbb{V}_{0}}+\left\langle\mathcal{F}\left(\Theta_{n}\right), \bar{\Theta}\right\rangle_{\mathbb{V}_{0}}=\delta\left\langle\theta_{n}^{K}, \bar{\phi}\right\rangle_{2}+\left\langle\mathcal{G}\left(\Theta_{n}\right), \bar{\Theta}\right\rangle_{\mathbb{V}_{0}}, \\
\left\langle\partial_{t} \theta_{n}^{K}, \bar{\theta}\right\rangle_{2}+\left\langle A_{K} \theta_{n}^{K}, \bar{\theta}\right\rangle_{2}+\left\langle\widehat{\mathcal{F}}\left(\theta_{n}^{K}\right), \bar{\theta}\right\rangle_{2}=-\delta\left\langle\partial_{t} \phi_{n}, \bar{\theta}\right\rangle_{2},
\end{array}\right.
$$

and

$$
\left\langle\Theta_{n}(0), \bar{\Theta}\right\rangle_{\mathbb{V}_{0}}=\left\langle\Theta_{n 0}, \bar{\Theta}\right\rangle_{\mathbb{V}_{0}},\left\langle\theta_{n}^{K}(0), \bar{\theta}\right\rangle_{2}=\left\langle\theta_{n 0}, \bar{\theta}\right\rangle_{2}
$$

for all $\bar{\Theta}=\left(\frac{\bar{\phi}}{\psi}\right) \in \mathcal{K}_{n}$, and all $\bar{\theta} \in \mathcal{P}_{n}^{K}$.

We aim to apply the standard existence theorems for ODE's. For this purpose, if $n$ is fixed, let us choose $\bar{\Theta}=\Theta_{j}$, and $\bar{\theta}=\theta_{j}^{K}, 1 \leq j \leq n$ and substitute the expressions (3.58) to the unknowns $\Theta_{n}$ and $\theta_{n}^{K}$ in (3.61). We seek to rewrite (3.61) in compact form. For this purpose, consider now the column vectors $\mathbf{d}=\left\{d_{i}(t)\right\}_{i \in \mathbf{N}}$ and $\mathbf{e}=\left\{e_{i}(t)\right\}_{i \in \mathbf{N}}$ and define the following matrices:

$$
\begin{gathered}
\mathbb{G}_{0}=\left(\left\langle\mathcal{N}\left(\begin{array}{c}
\phi_{i} \\
\psi_{i}
\end{array}\right),\left(\begin{array}{c}
\phi_{j} \\
\psi_{j}
\end{array}\right)\right\rangle_{\mathbf{V}_{0}}\right)_{1 \leq i, j \leq n}, \\
\mathbb{G}_{1}=\left(\left\langle\widehat{\lambda}_{i}\left(\begin{array}{c}
\phi_{i} \\
\psi_{i}
\end{array}\right),\left(\begin{array}{c}
\phi_{j} \\
\psi_{j}
\end{array}\right)\right\rangle_{\mathbf{V}_{0}}\right)_{1 \leq i, j \leq n}=\widehat{\lambda}_{i} \mathbb{I}_{n \times n}, \\
\mathbb{F}_{0}(\mathbf{d})=\left(\left\langle\left(\begin{array}{c}
F_{1}^{\prime}\left(\sum_{i=1}^{n} d_{i}(t) \phi_{i}\right) \\
F_{2}^{\prime}\left(\sum_{i=1}^{n} d_{i}(t) \psi_{i}\right)
\end{array}\right),\left(\begin{array}{c}
\phi_{j} \\
\psi_{j}
\end{array}\right)\right\rangle_{\mathbf{V}_{0}}\right)_{1 \leq i, j \leq n}, \\
\mathbb{W}_{0}=\left(\left\langle\theta_{i}^{K}, \phi_{j}\right\rangle_{2}\right)_{1 \leq i, j \leq n}, \mathbb{W}_{1}=\left(\left\langle\phi_{i}, \theta_{j}^{K}\right\rangle_{2}\right)_{1 \leq i, j \leq n}, \\
\mathbb{G}_{2}=\left(\left\langle\theta_{i}^{K}, \theta_{j}^{K}\right\rangle_{2}\right)_{1 \leq i, j \leq n}=\mathbb{I}_{n \times n}, \mathbb{G}_{3}=\left(\left\langle\lambda_{i}^{K} \theta_{i}^{K}, \theta_{j}^{K}\right\rangle_{2}\right)_{1 \leq i, j \leq n}=\lambda_{i}^{K} \mathbb{I}_{n \times n}, \\
\mathbb{G}_{4}=\left(|\Gamma|\left\langle\partial_{n} \phi_{i}\right\rangle_{\Gamma}\left\langle\phi_{j}\right\rangle\right)_{1 \leq i, j \leq n}, \mathbb{G}_{5}=\left(|\Omega|\left\langle\theta_{i}^{K}\right\rangle\left\langle\phi_{j}\right\rangle\right)_{1 \leq i, j \leq n},
\end{gathered}
$$

and

$$
\begin{gathered}
\mathbb{F}_{1}(\mathbf{e})=\left(\left\langle F_{3}^{\prime}\left(\sum_{i=1}^{n} e_{i}(t) \theta_{i}^{K}\right), \theta_{j}^{K}\right\rangle_{2}\right)_{1 \leq i, j \leq n} \\
\mathbb{F}_{2}(\mathbf{d})=\left(|\Omega|\left\langle F_{1}^{\prime}\left(\sum_{i=1}^{n} d_{i}(t) \phi_{i}\right)\right\rangle\left\langle\phi_{j}\right\rangle\right)_{1 \leq i, j \leq n}
\end{gathered}
$$


After performing direct calculations (and using the above definitions), we actually derive the following system of equations in compact form:

$$
\left\{\begin{array}{c}
\mathbb{G}_{0} \mathbf{d}^{\prime}(t)+\left(\mathbb{G}_{1}+\mathbb{G}_{4}\right) \mathbf{d}(t)+\mathbb{F}_{0}(\mathbf{d}(t))-\mathbb{F}_{2}(\mathbf{d}(t))=\delta\left(\mathbb{W}_{0}-\mathbb{G}_{5}\right) \mathbf{e}(t), \\
\mathbb{G}_{2} \mathbf{e}^{\prime}(t)+\mathbb{G}_{3} \mathbf{e}(t)+\mathbb{F}_{1}(\mathbf{e}(t))=-\delta \mathbb{W}_{1} \mathbf{d}^{\prime}(t) .
\end{array}\right.
$$

First, note that, based on our definitions, $\mathbb{G}_{2}$ is the identity matrix, whereas the matrix coefficient $\mathbb{G}_{0}$ of $\mathbf{d}^{\prime}$ in (3.63) is symmetric and positive-definite, hence, non-singular. Secondly, both matrices $\mathbb{G}_{1}$ and $\mathbb{G}_{3}$ are diagonal, with their diagonal entries being given by the eigenvalues $\left\{\widehat{\lambda}_{i}\right\}_{i}$ and $\left\{\lambda_{i}^{K}\right\}_{i}$, respectively. Besides, the bilinear forms $\left\langle\mathcal{B} \Theta_{i}, \Theta_{j}\right\rangle_{\mathbb{V}_{0}}=\left\langle\Theta_{i}, \Theta_{j}\right\rangle_{\mathbb{V}_{1}}=\widehat{\lambda}_{i}\left\langle\Theta_{i}, \Theta_{j}\right\rangle_{\mathbb{V}_{0}}$ and $\left\langle A_{K} \theta_{i}^{K}, \theta_{j}^{K}\right\rangle_{2}=$ $\lambda_{i}^{K}\left\langle\theta_{i}^{K}, \theta_{j}^{K}\right\rangle_{2}$ are $\mathbb{V}_{1}$-coercive and $L^{2}(\Omega)$-coercive, respectively. Then, computing for $\mathbf{d}^{\prime}$ from the first equation of (3.63) and replacing the expression that we obtain into the right-hand side of the second equation of (3.63), we can transform our problem and the initial conditions of $\left(\mathbb{P}_{n}^{K}\right)$ into a Cauchy problem for a system of nonlinear ordinary differential equations:

$$
\left\{\left(\begin{array}{c}
\partial_{t} \mathbf{e}(t) \\
\partial_{t} \mathbf{d}(t)
\end{array}\right)=\left(\begin{array}{l}
\mathbb{U}_{1}(t, \mathbf{d}(t), \mathbf{e}(t)) \\
\mathbb{U}_{2}(t, \mathbf{d}(t), \mathbf{e}(t))
\end{array}\right),\right.
$$

where $\mathbb{U}_{l}:\left[\tau, t_{n}\right] \times \mathbf{R}^{2 n} \rightarrow \mathbf{R}^{2 n}, l=1,2$, with

$$
\begin{gathered}
\mathbb{U}_{1}(t, \mathbf{d}, \mathbf{e})=\mathbb{G}_{3} \mathbf{e}+\mathbb{F}_{1}(\mathbf{e})+\delta \mathbb{W}_{1} \mathbb{G}_{0}^{-1}\left[\left(\mathbb{G}_{1}+\mathbb{G}_{4}\right) \mathbf{d}+\mathbb{F}_{0}(\mathbf{d})-\mathbb{F}_{2}(\mathbf{d})-\delta\left(\mathbb{W}_{0}-\mathbb{G}_{5}\right) \mathbf{e}\right] \\
\mathbb{U}_{2}(t, \mathbf{d}, \mathbf{e})=-\mathbb{G}_{0}^{-1}\left[\left(\mathbb{G}_{1}+\mathbb{G}_{4}\right) \mathbf{d}+\mathbb{F}_{0}(\mathbf{d})-\mathbb{F}_{2}(\mathbf{d})+\delta\left(\mathbb{W}_{0}-\mathbb{G}_{5}\right) \mathbf{e}\right]
\end{gathered}
$$

Each function $\mathbb{U}_{l}, l=1,2$ is continuous with respect to its arguments, uniformly in $t$, by the continuity of the projections and the fact that $F_{i} \in C(\mathbf{R})(i=1,2,3)$. Applying Cauchy's theorem for ODE's, we find a small time $t_{n} \in(0, T)$ such that (3.64) (and thus (3.63)) holds for all $t \in\left[0, t_{n}\right]$. This gives the desired local $C^{1}-$ solution $\Phi=(\phi, \psi, \theta)$ to our problem (3.63), since $\Phi_{n}=\left(\phi_{n}, \psi_{n}, \theta_{n}^{K}\right)$ satisfies (3.63). Now, based on the uniform a priori estimates with respect to $t$, derived for the solution $\Phi=(\phi, \psi, \theta)$ of $(2.4)-(2.10)$, we obtain, in particular, that any local solution of (3.63) is actually a global solution that is defined on the whole interval $[0, T]$. It remains then to pass to the limit as $n \rightarrow \infty$.

According to the a priori estimates derived in the beginning of Section 3, we have

$$
\begin{gathered}
\left\|\phi_{n}\right\|_{L^{\infty}\left([0, T] ; H^{2}(\Omega)\right)}+\left\|\psi_{n}\right\|_{L^{\infty}\left([0, T] ; H^{2}(\Gamma)\right)}+\left\|\theta_{n}\right\|_{L^{\infty}\left([0, T] ; H^{2}(\Omega)\right)} \leq C, \\
\left\|\partial_{t} \phi_{n}\right\|_{L^{2}\left([0, T] ; H^{1}(\Omega)\right)}+\left\|\partial_{t} \psi_{n}\right\|_{L^{2}\left([0, T] ; H^{1}(\Gamma)\right)}+\left\|\partial_{t} \theta_{n}\right\|_{L^{2}\left([0, T] ; L^{2}(\Omega)\right)} \leq C .
\end{gathered}
$$

The approximate chemical potentials $\mu_{n}, n \in \mathbf{N}$, satisfy

$$
\left\|\mu_{n}\right\|_{L^{\infty}\left([0, T] ; H^{2}(\Omega)\right)} \leq C
$$

where $C$ depends on $\Omega, \Gamma, T, \alpha, \delta, \phi_{0}, \psi_{0}, \theta_{0}$ but is independent of $n$ and $t$. From this point on, all convergence relations will be intended to hold up to the extraction of suitable subsequences, generally not relabelled. Thus, we observe that weak and weak star compactness results applied to the above sequences $\Phi_{n}=\left(\phi_{n}, \psi_{n}, \theta_{n}\right)$ entail that there exist the function $\Phi=(\phi, \psi, \theta)$ such that as $n \rightarrow \infty$, the following properties hold:

$$
\begin{gathered}
\Phi_{n} \rightarrow \Phi \text { weakly star in } L^{\infty}\left([0, T] ; H^{2}(\Omega) \times H^{2}(\Gamma) \times H^{2}(\Omega)\right), \\
\left(\partial_{t} \phi_{n}, \partial_{t} \psi_{n}\right) \rightarrow\left(\partial_{t} \phi, \partial_{t} \psi\right) \text { weakly in } L^{2}\left([0, T] ; H^{1}(\Omega) \times H^{1}(\Gamma)\right),
\end{gathered}
$$




$$
\begin{gathered}
\partial_{t} \theta_{n} \rightarrow \partial_{t} \theta \text { weakly in } L^{2}\left([0, T] ; L^{2}(\Omega)\right), \\
\mu_{n} \rightarrow \mu \text { weakly star in } L^{\infty}\left([0, T] ; H^{2}(\Omega)\right) .
\end{gathered}
$$

Then, standard interpolation (for instance, $H^{2-\eta}(\Omega) \subset L^{\infty}(\Omega)$, for $\eta \in(0,1 / 2)$, since $\Omega \subset \mathbf{R}^{n}$ with $n=3$ ) and compact embedding results for vector valued functions (see e.g., [22, Lemma 8]) ensure that

$$
\Phi_{n} \rightarrow \Phi \text { strongly in } C([0, T] ; C(\Omega) \times C(\Gamma) \times C(\Omega)) .
$$

Standard arguments and (3.65) imply that $\Phi(0)=\Phi_{0}$. By the Lipschitz continuity of the nonlinear functions $F_{i}^{\prime}, i=1,2,3$, and standard compactness results (see e.g., [22, Theorem 9]), the above convergence allows us to infer that

$$
\begin{aligned}
& F_{1}^{\prime}\left(\phi_{n}\right) \rightarrow F_{1}^{\prime}(\phi) \text { strongly in } C\left([0, T] ; L^{2}(\Omega)\right), \\
& F_{2}^{\prime}\left(\psi_{n}\right) \rightarrow F_{2}^{\prime}(\psi) \text { strongly in } C\left([0, T] ; L^{2}(\Gamma)\right),
\end{aligned}
$$

and

$$
F_{3}^{\prime}\left(\theta_{n}\right) \rightarrow F_{3}^{\prime}(\theta) \text { strongly in } C\left([0, T] ; L^{2}(\Omega)\right) .
$$

Thus, passing to the limit in (3.61) - (3.62) and using the above convergence properties, we immediately have that the solution $\Phi=(\phi, \psi, \theta)$ satisfies $(3.1)-(3.3)$ (and therefore $(2.4)-(2.10)$ ) in the sense introduced in Definition 1, Section 2. It also worth mentioning that since $\left(\partial_{t} \phi, \partial_{t} \psi\right) \in L^{2}\left([0, T] ; \mathbb{V}_{1}\right)$, by regularity of elliptic equations (cf. estimate (3.42), see also $[\mathbf{4 1}]$ ) for the boundary value problem (3.41), we also deduce that $\phi \in L^{2}\left([0, T] ; H^{3}(\Omega)\right)$ and $\psi \in L^{2}\left([0, T] ; H^{3}(\Omega)\right)$. Finally, using this new regularity and a standard result from elliptic theory applied to equations (2.4), (2.7), we obtain that $\mu \in L^{2}\left([0, T] ; H^{3}(\Omega)\right)$, as well.

We conclude this section with the following result on the solvability of our problem $(2.4)-(2.10)$. Let $T>0$ be fixed, but otherwise arbitrary.

Corollary 7. Let $\left(\phi_{0}, \psi_{0}, \theta_{0}\right) \in \mathbb{Y}_{\alpha}^{K}, K \in\{D, N, R\}$ and suppose that the nonlinearities $F_{i}, i=1,2,3$ satisfy assumptions $(1.12)-(1.14)$. Then, problem $(2.4)-(2.10)$ has a unique solution $(\phi(t), \psi(t), \theta(t))$ that belongs to the space $C\left([0, T], \mathbb{Y}_{\alpha}^{K}\right) \cap L_{\text {loc }}^{\infty}\left((0, T], \mathbb{Z}_{3}\right)$ and satisfies $(3.52)$. Moreover, this problem defines a semigroup $\mathcal{S}_{t}^{K}$ in the phase space $\mathbb{Y}_{\alpha}^{K}$ by

$$
\mathcal{S}_{t}^{K}: \mathbb{Y}_{\alpha}^{K} \rightarrow \mathbb{Y}_{\alpha}^{K}
$$

such that

$$
\mathcal{S}_{t}^{K}\left(\phi_{0}, \psi_{0}, \theta_{0}\right)=(\phi(t), \psi(t), \theta(t)) .
$$

Proof. The uniqueness of a solution was actually verified in Theorem 6 and the existence of solutions in the phase space $\mathbb{Y}_{\alpha}^{K}$ was verified above using a FaedoGalerkin approximation scheme. Thus, problem $(2.4)-(2.10)$ generates a semiflow $\mathcal{S}_{t}^{K}$ on $\mathbb{Y}_{\alpha}^{K}$ given by $(3.66)$, where $(\phi(t), \psi(t), \theta(t))$ solves (2.4)-(2.10) with initial data in $\mathbb{Y}_{\alpha}^{K}$. The corollary is proved.

\section{Global and exponential attractors}

We are finally ready to construct an exponential attractor $\mathcal{M}^{K}$ in $\mathbb{Y}_{\alpha}^{K}$ for our problem. Let us mention that due to equations (2.4) and (2.7), our system possesses a conservation law (2.1), so that (2.4) - (2.10) is not dissipative on the entire phase space $\mathbb{Y}_{\alpha}^{K}$, so then we have to restrict ourselves to the strips

$$
\mathbb{Y}_{\alpha}^{K, m}:=\mathbb{Y}_{\alpha}^{K} \cap\{|\langle\phi\rangle| \leq m\}
$$


for any given $m>0$. Precisely, our main results of this section are stated below.

THEOREM 8. Suppose that the nonlinearities $F_{i} \in C^{2}(\mathbf{R}), i=1,2$, satisfy the assumptions (1.12)-(1.14). For every $K \in\{D, N, R\}$, there is a connected compact global attractor $\mathcal{A}_{K} \subset \mathbb{Y}_{\alpha}^{K, m} \cap \mathbb{Z}_{3}$ for the semi-flow $S_{t}^{K}$ associated to the system of equations (2.4) - (2.10). Moreover, $\mathcal{A}_{K}$ has finite fractal dimension.

Proof. Theorem 4 and 5 imply that the dynamical system $\left(\mathbb{Y}_{\alpha}^{K, m}, \mathcal{S}_{t}^{K}\right)$ has a bounded absorbing set and a compact absorbing set $\mathbb{B}_{0}$ which is contained in $\mathbb{V}_{3} \times H^{3}(\Omega)$. Therefore, recalling that $\mathcal{S}_{t}^{K}$ is also a closed semigroup (cf. Theorem $6,(3.52))$, the proof follows from [47, Theorem 2 and Corollary 6] (see also [23]).

The finite dimensionality of the attractor $\mathcal{A}_{K}$ will be a consequence of the theorem below.

TheOREm 9. Let $K \in\{D, N, R\}$ be fixed. Let the nonlinearities $F_{i} \in C^{2,1}(\mathbf{R})$ satisfy the assumptions $(1.12)-(1.14)$. Then, there exists a compact set $\mathcal{M}^{K, m} \subset$ $\mathbb{Y}_{\alpha}^{K, m} \cap \mathbb{Z}_{3}$, which satisfies the following:

(i) $\mathcal{M}^{K, m}$ is semi-invariant with respect to the semiflow $\mathcal{S}_{t}^{K}$ associated with problem $(2.4)-(2.10)$, that is,

$$
\mathcal{S}_{t}^{K}\left(\mathcal{M}^{K, m}\right) \subset \mathcal{M}^{K, m}, t \geq 0 .
$$

(ii) The fractal dimension of $\mathcal{M}^{K, m}$ is finite, that is,

$$
\operatorname{dim}_{F}\left(\mathcal{M}^{K, m}, \mathbb{Z}_{2}\right) \leq C<+\infty
$$

where $C$ can be determined explicitly in terms of the given parameters (see e.g., [15]).

(iii) $\mathcal{M}^{K, m}$ attracts exponentially fast the bounded subsets of $\mathbb{Y}_{\alpha}^{K, m}$, that is, there exists a constant $\rho>0$ and a monotonic function $Q_{\alpha}$ independent of $t$ such that

$$
\operatorname{dist}_{\mathbb{Z}_{2}}\left(\mathcal{S}_{t}^{K} B, \mathcal{M}^{K, m}\right) \leq Q_{\alpha}\left(\|B\|_{\mathbb{Y}_{\alpha}^{K, m}}\right) e^{-\rho t}, t \geq 0,
$$

for every bounded subset $B$ of $\mathbb{Y}_{\alpha}^{K, m}$. Here dist $\mathbb{Z}_{2}$ denotes the non-symmetric Hausdorff distance between sets in $\mathbb{Z}_{2}$.

Obviously, the exponential attractor $\mathcal{M}^{K, m}$ and the semigroup $\mathcal{S}_{t}^{K}$ depend on $m$. To prove our last theorem, we introduce the following ball $\mathbb{B}^{K, m}$ with sufficiently large radius $R$ in the space $\mathbb{Y}_{\alpha}^{K, m}$ :

$$
\mathbb{B}_{R}^{K, m}:=\left\{\Phi=(\phi, \psi, \theta) \in \mathbb{Y}_{\alpha}^{K, m},\|\Phi\|_{\mathbb{Y}_{\alpha}^{K, m}} \leq R,|\langle\phi\rangle| \leq m\right\} .
$$

Due to the dissipative estimate (3.20) and the smoothing property (3.34), there exist sufficiently large $\bar{R}=\bar{R}(m)$ and $t^{\#}=t^{\#}(\bar{R}, m)$ which are independent of $t$ such that $\mathbb{B}^{K, m}:=\mathbb{B}_{\frac{K}{R}}^{K, m}$ is an absorbing set for the semigroup $\mathcal{S}_{t}^{K}$ acting on $\mathbb{Y}_{\alpha}^{K, m}$ and

$$
\mathcal{S}_{t}^{K}\left(\mathbb{B}^{K, m}\right) \subset \mathbb{B}^{K, m}, \text { for } t \geq t^{\#} .
$$

Before we construct an exponential attractor to our problem, we will need an additional lemma. We will derive a smoothing estimate for the difference of two solutions of $(2.4)-(2.10)$. Let $K \in\{D, N, R\}$ be fixed. 
Lemma 10. Let $\left(\phi_{1}, \psi_{1}, \theta_{1}\right)$ and $\left(\phi_{2}, \psi_{2}, \theta_{2}\right)$ be two solutions of $(2.4)-(2.10)$ with initial data $\left(\phi_{i}(0), \psi_{i}(0), \theta_{i}(0)\right), i=1,2$, in $\mathbb{B}_{R}^{K, m}$, for given $R>0$. Then, for every $t>0$, we have the following smoothing estimate:

$$
\leq C e^{L t} \frac{t+1}{t}\left(\left\|\phi_{1}(0)-\phi_{2}(0)\right\|_{H^{1}}^{2}+\left\|\psi_{1}(0)-\psi_{2}(0)\right\|_{H^{1}(\Gamma)}^{2}+\left\|\theta_{1}(0)-\theta_{2}(0)\right\|_{2}^{2}\right),
$$

$$
\left\|\phi_{1}(t)-\phi_{2}(t)\right\|_{H^{2}}^{2}+\left\|\psi_{1}(t)-\psi_{2}(t)\right\|_{H^{2}(\Gamma)}^{2}+\left\|\theta_{1}(t)-\theta_{2}(t)\right\|_{Z_{K}}^{2}
$$

where $C, L$ depend only on $\alpha$ and $R$, but they are independent of $t$.

Proof. Set

$$
(\bar{\phi}, \bar{\psi}, \bar{\theta}, \mu):=\left(\phi_{1}-\phi_{2}, \psi_{1}-\psi_{2}, \theta_{1}-\theta_{2}, \mu_{1}-\mu_{2}\right) .
$$

Recall that the functions $\bar{\phi}, \bar{\psi}, \bar{\theta}$ and $\mu$ satisfy (3.53), (3.54). Differentiating now all equations of (3.53) with respect to $t$ (recall that we can do so within an appropriate Faedo-Galerkin scheme), multiplying the first, third and fourth scalarly by $A_{N}^{-1} \partial_{t} \bar{\phi}(t), \partial_{t} \bar{\phi}(t)$ and $\partial_{t} \bar{\psi}(t)$, respectively, summing up the relations that we obtain with the first equation of (3.54) multiplied by $\partial_{t} \bar{\theta}(t)$, and integrating by parts, we obtain after standard transformations:

$$
\begin{gathered}
\frac{d}{d t}\left[\alpha\left\|\partial_{t} \bar{\phi}(t)\right\|_{2}^{2}+\left\|\partial_{t} \bar{\phi}(t)\right\|_{H^{-1}}^{2}+\left\|\partial_{t} \bar{\psi}(t)\right\|_{2, \Gamma}^{2}+\left\langle A_{K} \bar{\theta}(t), \bar{\theta}(t)\right\rangle_{2}\right] \\
+2\left\|\partial_{t} \bar{\phi}(t)\right\|_{H^{1}}^{2}+2\left\|\partial_{t} \bar{\psi}(t)\right\|_{H^{1}(\Gamma)}^{2}+2\left\|\partial_{t} \bar{\theta}(t)\right\|_{2}^{2} \\
=-2\left\langle l_{1}(t) \partial_{t} \bar{\phi}(t), \partial_{t} \bar{\phi}(t)\right\rangle_{2}-2\left\langle\partial_{t} l_{1}(t) \bar{\phi}(t), \partial_{t} \bar{\phi}(t)\right\rangle_{2}-2\left\langle l_{3}(t) \bar{\theta}(t), \partial_{t} \bar{\theta}(t)\right\rangle_{2} \\
(4.8) \quad-2\left\langle l_{2}(t) \partial_{t} \bar{\psi}(t), \partial_{t} \bar{\psi}(t)\right\rangle_{2, \Gamma}-2\left\langle\partial_{t} l_{2}(t) \bar{\psi}(t), \partial_{t} \bar{\psi}(t)\right\rangle_{2, \Gamma} .
\end{gathered}
$$

The first and fourth term on the right-hand side of (4.8) can be estimated using (3.55), and for the remaining terms, we use the embeddings $H^{1}(\Omega) \subset L^{4}(\Omega)$, $H^{1}(\Gamma) \subset L^{4}(\Gamma)$ so that we have

$$
\begin{gathered}
\frac{d}{d t}\left[\alpha\left\|\partial_{t} \bar{\phi}(t)\right\|_{2}^{2}+\left\|\partial_{t} \bar{\phi}(t)\right\|_{H^{-1}}^{2}+\left\|\partial_{t} \bar{\psi}(t)\right\|_{2, \Gamma}^{2}+\left\langle A_{K} \bar{\theta}(t), \bar{\theta}(t)\right\rangle_{2}\right] \\
+2\left\|\partial_{t} \bar{\phi}(t)\right\|_{H^{1}}^{2}+2\left\|\partial_{t} \bar{\psi}(t)\right\|_{H^{1}(\Gamma)}^{2}+2\left\|\partial_{t} \bar{\theta}(t)\right\|_{2}^{2} \\
\leq L\left[\alpha\left\|\partial_{t} \bar{\phi}(t)\right\|_{2}^{2}+\left\|\partial_{t} \bar{\psi}(t)\right\|_{2, \Gamma}^{2}\right]+2\left\|\partial_{t} l_{1}(t)\right\|_{2}\left\|\bar{\phi}(t) \partial_{t} \bar{\phi}(t)\right\|_{2} \\
+2\left\|\partial_{t} l_{2}(t)\right\|_{2, \Gamma}\left\|\bar{\psi}(t) \partial_{t} \bar{\psi}(t)\right\|_{2, \Gamma}+2\left\|l_{3}(t) \bar{\theta}(t)\right\|_{2}\left\|\partial_{t} \bar{\theta}(t)\right\|_{2} \\
\leq L\left[\alpha\left\|\partial_{t} \bar{\phi}(t)\right\|_{2}^{2}+\left\|\partial_{t} \bar{\psi}(t)\right\|_{2, \Gamma}^{2}\right]+2 L^{\prime}\left[\|\bar{\phi}(t)\|_{4}\left\|\partial_{t} \bar{\phi}(t)\right\|_{4}+\|\bar{\psi}(t)\|_{4, \Gamma}\left\|\partial_{t} \bar{\psi}(t)\right\|_{4, \Gamma}\right] \\
+L\|\bar{\theta}(t)\|_{2}^{2}+\left\|\partial_{t} \bar{\theta}(t)\right\|_{2}^{2} \\
\leq L\left[\alpha\left\|\partial_{t} \bar{\phi}(t)\right\|_{2}^{2}+\left\|\partial_{t} \bar{\psi}(t)\right\|_{2, \Gamma}^{2}\right]+\left(\left\|\partial_{t} \bar{\phi}(t)\right\|_{H^{1}}^{2}+\left\|\partial_{t} \bar{\psi}(t)\right\|_{H^{1}(\Gamma)}^{2}+\left\|\partial_{t} \bar{\theta}(t)\right\|_{2}^{2}\right) \\
+L^{\prime \prime}\left(\|\bar{\phi}(t)\|_{H^{1}}^{2}+\|\bar{\psi}(t)\|_{H^{1}(\Gamma)}^{2}+\|\bar{\theta}(t)\|_{2}^{2}\right),
\end{gathered}
$$

where $L^{\prime}$ and $L^{\prime \prime}$ depend on $L$ and thus on the norm of the initial data in $\mathbb{B}_{R}^{K, m}$ and $\alpha$, but they are independent of $t$. Multiplying now both sides of (4.9) by $t$, we deduce

$$
\frac{d}{d t}\left[t\left(\alpha\left\|\partial_{t} \bar{\phi}(t)\right\|_{2}^{2}+\left\|\partial_{t} \bar{\phi}(t)\right\|_{H^{-1}}^{2}+\left\|\partial_{t} \bar{\psi}(t)\right\|_{2, \Gamma}^{2}+\left\langle A_{K} \bar{\theta}(t), \bar{\theta}(t)\right\rangle_{2}\right)\right]
$$




$$
\begin{gathered}
+t\left(\left\|\partial_{t} \bar{\phi}(t)\right\|_{H^{1}}^{2}+\left\|\partial_{t} \bar{\psi}(t)\right\|_{H^{1}(\Gamma)}^{2}+\left\|\partial_{t} \bar{\theta}(t)\right\|_{2}^{2}\right) \\
\leq L t\left[\alpha\left\|\partial_{t} \bar{\phi}(t)\right\|_{2}^{2}+\left\|\partial_{t} \bar{\psi}(t)\right\|_{2, \Gamma}^{2}\right]+L^{\prime \prime} t\left(\|\bar{\phi}(t)\|_{H^{1}}^{2}+\|\bar{\psi}(t)\|_{H^{1}(\Gamma)}^{2}+\|\bar{\theta}(t)\|_{2}^{2}\right) \\
+\alpha\left\|\partial_{t} \bar{\phi}(t)\right\|_{2}^{2}+\left\|\partial_{t} \bar{\phi}(t)\right\|_{H^{-1}}^{2}+\left\|\partial_{t} \bar{\psi}(t)\right\|_{2, \Gamma}^{2}+\left\langle A_{K} \bar{\theta}(t), \bar{\theta}(t)\right\rangle_{2} .
\end{gathered}
$$

Integrating (4.10) over $(0, t)$ and then employing estimate (3.52), we easily obtain that

$$
\begin{gathered}
\alpha\left\|\partial_{t} \bar{\phi}(t)\right\|_{2}^{2}+\left\|\partial_{t} \bar{\phi}(t)\right\|_{H^{-1}}^{2}+\left\|\partial_{t} \bar{\psi}(t)\right\|_{2, \Gamma}^{2}+\left\langle A_{K} \bar{\theta}(t), \bar{\theta}(t)\right\rangle_{2} \\
\leq C e^{L t} \frac{t+1}{t}\left(\|\bar{\phi}(0)\|_{H^{1}}^{2}+\|\bar{\psi}(0)\|_{H^{1}(\Gamma)}^{2}+\|\bar{\theta}(0)\|_{2}^{2}\right) .
\end{gathered}
$$

The estimate (4.7) is then a consequence of (4.11), standard parabolic arguments and maximum principles as in the proof of Theorem 4 (see $(3.26)-(3.33)$ ).

In order to prove Theorem 9, we use the method introduced in [15] (cf. also $[\mathbf{1 6}]$ ). We construct the required exponential attractor for the semigroup $\mathcal{S}_{t}^{K}$ with discrete times and extend the results to continuous times. We are ready to prove the existence of the following "discrete" exponential attractor $\widehat{\mathcal{M}}^{K, m}$ by using the following fundamental result on discrete semigroups (see [15]), which is reported below for the reader's convenience.

Theorem 11. Let $\mathcal{V}$ and $\mathcal{W}$ be two Banach spaces such that $\mathcal{W}$ is compactly embedded in $\mathcal{V}$. Let $X$ be a bounded subset of $\mathcal{W}$ and consider a nonlinear map $\Sigma: X \rightarrow X$ satisfying the smoothing property

$$
\left\|\Sigma\left(x_{1}\right)-\Sigma\left(x_{2}\right)\right\|_{\mathcal{W}} \leq C\left\|x_{1}-x_{2}\right\|_{\mathcal{V}},
$$

for all $x_{1}, x_{2} \in X$, where $C>0$ depends on $X$. Then the discrete dynamical system $\left(X, \Sigma^{n}\right)$ possesses a discrete exponential attractor $\mathcal{M}^{*} \subset \mathcal{V}$, that is, a compact set with finite fractal dimension such that

$$
\begin{gathered}
\Sigma\left(\mathcal{M}^{*}\right) \subset \mathcal{M}^{*}, \\
\operatorname{dist}_{\mathcal{V}}\left(\Sigma^{n}(B), \mathcal{M}^{*}\right) \leq C_{X} e^{-\rho_{*} n}, \quad n \in \mathbb{N},
\end{gathered}
$$

where $C_{X}$ and $\rho_{*}$ are positive constants independent of $n$, with the former depending on $X$.

Proof of Theorem 9. Using Theorems 4, 5, 6 and Lemma 10, (4.7), we can find a bounded subset $X$ of $\mathbb{Y}_{\alpha}^{K, m} \cap \mathbb{Z}_{3}$ and $t^{\#}>0$ such that, setting $\mathcal{W}:=$ $\mathbb{V}_{2} \times Z_{K}, \mathcal{V}:=\mathbb{V}_{1} \times L^{2}(\Omega)$ and $\Sigma=\mathcal{S}_{t \#}^{K}$, the mapping $\Sigma: X \rightarrow X$ enjoys the smoothing property (4.12). Therefore Theorem 11 applies to $\Sigma$ and there exists a compact set $\widehat{\mathcal{M}}^{K, m} \in X$ of finite fractal dimension (with respect to the metric in $\left.\mathcal{V}=\mathbb{V}_{1} \times L^{2}(\Omega)\right)$ that satisfies (4.13) and (4.14). Hence, setting as usual

$$
\mathcal{M}^{K, m}=\cup_{t \in[t \#, 2 t \#]} \mathcal{S}_{t}^{K} \widehat{\mathcal{M}}^{K, m},
$$

we have that (4.2) and (4.4) are fulfilled, however with the metric of $\mathbb{Z}_{2}$ being replaced by that of $\mathbb{V}_{1} \times L^{2}(\Omega)$. Finally, there only remains to verify that $\mathcal{M}^{K, m}$ has finite fractal dimension. We will show that the map $\mathcal{S}_{t}^{K}$ is uniformly Hölder continuous on $\left[t_{0}, T\right] \times \mathbb{B}^{K, m}$ in the metric of $\mathcal{V}$. It will then follow that $\mathcal{M}^{K, m}$ is still a compact set with finite fractal dimension such that

$$
\operatorname{dim}_{F}\left(\mathcal{M}^{K, m}, \mathcal{V}\right) \leq \operatorname{dim}_{F}\left(\widehat{\mathcal{M}}^{K, m}, \mathcal{V}\right)+2 .
$$


LEMMA 12. Under the assumptions of Lemma 10, every solution $(\phi(t), \psi(t), \theta(t))$ of problem (2.4) - (2.10) is Hölder continuous with respect to $t$, that is, for every $t \geq t_{0}>0$ and $0 \leq s \leq 1$, we have

$$
\begin{gathered}
\|\phi(t+s)-\phi(t)\|_{H^{1}}+\|\psi(t+s)-\psi(t)\|_{H^{1}(\Gamma)} \\
+\left\|\theta(t+s)-\theta_{2}(t)\right\|_{2} \leq Q\left(\|\phi(0), \psi(0), \theta(0)\|_{\mathbb{Y}_{\alpha}^{K}}\right) s^{1 / 2} .
\end{gathered}
$$

Proof. The proof is a direct corollary of the estimates proved in Section 3. Recall that, for every $t \in[0, T]$, with $T$ fixed, but otherwise arbitrary, we have (4.17)

$$
\int_{0}^{T+1}\left[\left\|\partial_{t} \phi(s)\right\|_{H^{1}}^{2}+\left\|\partial_{t} \psi(s)\right\|_{H^{1}(\Gamma)}^{2}+\left\|\partial_{t} \theta(s)\right\|_{2}^{2}\right] d s \leq Q\left(\|\phi(0), \psi(0), \theta(0)\|_{\mathbb{Y}_{\alpha}^{K}}\right):=\bar{L} .
$$

Moreover, for every $t \geq t_{0}>0$ and $0 \leq s \leq 1$, we infer from (4.17) that

$$
\begin{gathered}
\|\phi(t+s)-\phi(t)\|_{H^{1}}+\|\psi(t+s)-\psi(t)\|_{H^{1}(\Gamma)}+\left\|\theta(t+s)-\theta_{2}(t)\right\|_{2} \\
=\left\|\int_{t}^{t+s} \partial_{t} \phi(\tau) d \tau\right\|_{H^{1}}+\left\|\int_{t}^{t+s} \partial_{t} \psi(\tau) d \tau\right\|_{H^{1}(\Gamma)}+\left\|\int_{t}^{t+s} \partial_{t} \theta(\tau) d \tau\right\|_{2} \\
\leq \int_{t}^{t+s}\left(\left\|\partial_{t} \phi(\tau)\right\|_{H^{1}}+\left\|\partial_{t} \psi(\tau)\right\|_{H^{1}(\Gamma)}+\left\|\partial_{t} \theta(\tau)\right\|_{2}\right) d \tau \leq C|s|^{1 / 2} .
\end{gathered}
$$

Thus, the lemma is proved.

In order to obtain the finite-dimentionality (4.3) and the required exponential convergence (4.4) of $\mathcal{M}^{K, m}$ in the required metric of $\mathbb{Z}_{2}$, it remains to recall that $\mathcal{S}_{t}^{K}, t \geq t_{0}$, possesses the smoothing property (3.34) and to use the standard interpolation inequalities $\|\cdot\|_{H^{2}} \leq C\|\cdot\|_{2}^{1 / 3}\|\cdot\|_{H^{3}}^{2 / 3},\|\cdot\|_{H^{2}} \leq C\|\cdot\|_{Z_{K}}^{1 / 2}\|\cdot\|_{H^{3}}^{1 / 2}$, where the constant $C$ is independent of $t$. The proof of Theorem 9 is now complete.

REMARK 13. Let us say more about the structure of the global attractor $\mathcal{A}_{K}$. But, first suppose that $F_{3} \equiv 0$ in (2.6). Then, in addition to the mass enthalpy condition (2.1), we also have conservation of temperature $\langle\theta\rangle=\left\langle\theta_{0}\right\rangle$, when $K=N$, due to the equations (2.6) and (2.9). Let us set

$$
\begin{aligned}
\mathcal{L}_{K}\left(\phi_{0}, \psi_{0}, \theta_{0}\right) & =\frac{1}{2}\left(\left\|\nabla \phi_{0}\right\|_{2}^{2}+\left\|\nabla_{\Gamma} \psi_{0}\right\|_{2, \Gamma}^{2}+\left\|\psi_{0}\right\|_{2, \Gamma}^{2}+\left\|\theta_{0}\right\|_{2}^{2}\right) \\
& +\int_{\Omega} F_{1}\left(\phi_{0}\right) d x+\int_{\Gamma} F_{2}\left(\psi_{0}\right) d S,
\end{aligned}
$$

for all $\left(\phi_{0}, \psi_{0}, \theta_{0}\right) \in \mathbb{Y}_{\alpha}^{K}$, if $K \in\{D, N, R\}$. Then, it is not difficult to prove that $\mathcal{L}_{K}$ is a (global) Lyapunov functional for the semi-flow $\mathcal{S}_{t}^{K}$ (cf. e.g. [46]). We can verify that for all $t>0$, in fact, we have

$$
\begin{gathered}
\frac{d}{d t} \mathcal{L}_{K}\left(\mathcal{S}_{t}^{K}\left(\phi_{0}, \psi_{0}, \theta_{0}\right)\right)=-\|\nabla \mu(t)\|_{2}^{2}-\left\|\partial_{t} \phi(t)\right\|_{2}^{2}-\left\|\partial_{t} \psi(t)\right\|_{2, \Gamma}^{2}-\|\nabla \theta(t)\|_{2}^{2} \\
-\frac{c}{b}\left\|\theta_{\mid \Gamma}(t)\right\|_{2, \Gamma}^{2} .
\end{gathered}
$$

Hence our semi-flow is a gradient system and this entails that $\mathcal{A}_{K}$ coincides with the unstable manifold of the set $\mathcal{E}_{K}$ of the stationary points (cf., e.g., [51, Chapter 
7, Section 4]). Notice that $\left(\phi_{\infty}, \psi_{\infty}, \theta_{\infty}\right) \in \mathbb{Y}_{\alpha}^{K}$ belongs to $\mathcal{E}_{K}$ if and only if it is a solution to the boundary value problem

$$
\begin{gathered}
-\Delta \phi_{\infty}+F_{1}^{\prime}\left(\phi_{\infty}\right)-\delta \theta_{\infty}=\text { const, } \\
-\Delta \theta_{\infty}=0, \quad \text { in } \Omega, \\
-\Delta_{\Gamma} \psi_{\infty}+\partial_{n} \phi_{\infty}+\psi_{\infty}+F_{2}^{\prime}\left(\psi_{\infty}\right)=0, \quad \text { on } \Gamma, \\
b \partial_{\mathbf{n}} \theta_{\infty}+c \theta_{\infty}=0, \quad \text { on } \Gamma .
\end{gathered}
$$

Also, for any triplet of initial data $\left(\phi_{0}, \psi_{0}, \theta_{0}\right) \in \mathbb{Y}_{\alpha}^{K}$, the functional $\mathcal{L}_{K}$ is constant on $\omega\left(\phi_{0}, \psi_{0}, \theta_{0}\right)$ and, since each trajectory originated from $\mathbb{Y}_{\alpha}^{K}$ is precompact (see Theorem 5), it follows that $\omega\left(\phi_{0}, \psi_{0}, \theta_{0}\right)$ is a connected and compact invariant set which is contained in $\mathcal{E}_{K}$ (see [56, Chapter 6, Section 6.3]). This set can be a continuum, therefore each trajectory might not converge to a single equilibrium. However, if $F_{i}, i=1,2$ are real analytic, it should be possible to prove this kind of convergence via a suitable adaptation of the Eojasiewicz-Simon inequality. This result would be a generalization of [46, Theorem 5.5]. We shall investigate this problem in a forthcoming article.

ACKNowledgement 14. I want to thank professor Alain Miranville for his suggestions and remarks that have subsequently improved the article. The author also wishes to thank the referee for his/her careful reading of the paper and his/her many useful comments.

\section{References}

[1] S. M. Allen, J.W. Cahn, A microscopic theory for the antiphase boundary motion and its application to antiphase domain coarsening, Acta Metallurgica 27 (1979), 1085-1095.

[2] S. Aizicovici, H. Petzeltova, Asymptotic behaviour of solutions of a conserved phase-field system with memory, Journal of Integral Eqn. and Appl. 15 (2003), 217-240.

[3] D. Brochet, X. Chen, D. Hilhorst, Finite dimensional exponential attractor for the phase-field model, Appl. Anal. 49 (1993), 197-212.

[4] D. Brochet, D. Hilhorst, Universal attractor and inertial sets for the phase-field model, Appl. Math. Lett. 4 (1991), 59-62.

[5] M. Brokate, J. Sprekels, Hysteresis and Phase Transitions, Springer, New York, 1996.

[6] P. W. Bates, S. Zheng, Inertial manifolds and inertial sets for the phase-field equations, J. Dynamics Differential Equations 4 (1992), 375-397.

[7] G. Caginalp, An analysis of a phase field model of a free boundary, Arch. Ration. Mech. Anal. 92 (1986), 205-245.

[8] The dynamics of a conserved phase-field system: Stefan-like, Hele-Shaw, and Cahn-Hilliard models as asymptotic limits, IMA J. Appl. Math. 44 (1990), 77-94.

[9] R. Chill, E. Fašangovà, J. Prüss: Convergence to steady states of solutions of the CahnHilliard and Caginalp equations with dynamic boundary conditions, Math. Nachr. 13 (2006), 1448-1462.

[10] P. Colli, G. Gilardi, M. Grasselli, G. Schimperna, Global existence for the conserved phase field model with memory and quadratic nonlinearity, Portugaliae Mathematica, vol. 58 (2001), 159-170.

[11] P. Colli, P. Laurencot, Uniqueness of weak solutions to the phase-field model with memory, J. Math. Sci. Univ. Tokyo 5 (1998), 459-476.

[12] J. W. Cahn, E. Hilliard, Free energy of a nonuniform system, J. Chem. Phys. 28 (1958), 258-367.

[13] L. Cherfils, A. Miranville, Some remarks on the asymptotic behavior of the Caginalp system with singular potentials, Adv. Math. Sci. Appl. 16 (2007), 107-129.

[14] A. Damlamian, N. Kenmochi, N. Sato, Subdifferential operator approach to a class of nonlinear systems for Stefan problems with phase relaxation, Nonlinear Anal. 23 (1994), 115-142.

[15] M. Efendiev, A. Miranville, S. Zelik, Exponential attractors for a nonlinear reaction-diffusion system in $\mathbb{R}^{3}$, C. R. Math. Acad. Sci. Paris 330 (2000), 713-718. 
[16] M. Efendiev, A. Miranville, S. Zelik, Exponential attractors for a singularly perturbed CahnHilliard system, Math. Nachr. 272 (2004), 11-31.

[17] C. M. Elliott, S. Zheng, Global existence and stability of solutions to the phase-field equations, in "Free boundary problems", Internat. Ser. Numer. Math. 95 (1990), 46-58, Birkhäuser Verlag, Basel.

[18] C. M. Elliott and S. Zheng, On the Cahn-Hilliard equation, Arch. Rational Mech. Anal. 96, (1986), 339-357.

[19] G. B. McFadden, Phase-field models of solidification, Contemp. Math. 306 (2002), 107-145.

[20] H. P. Fischer, Ph. Maass, W. Dieterich, Novel surface modes of spinodal decomposition, Phys. Rev. Letters 79 (1997), 893-896.

[21] H. P. Fischer, Ph. Maass, W. Dieterich, Diverging time and length scales of spinodal decomposition modes in thin flows, Europhys. Letters 62 (1998), 49-54.

[22] C. G. Gal, Global well-posedness for a non-isothermal Cahn-Hilliard model with dynamic boundary conditions, Advances in Differential Equations 12 (2007), 1241-1274.

[23] C. G. Gal, M. Grasselli, The non-isothermal Allen-Cahn equation with dynamic boundary conditions, Discrete and Continuous Dynamical Systems, to appear.

[24] C. G. Gal, M. Grasselli, A. Miranville, Robust exponential attractors for singularly perturbed phase-field equations with dynamic boundary conditions, Nonlinear Differential Equations and Applications, to appear.

[25] C. G. Gal, G. R. Goldstein, J. A. Goldstein, S. Romanelli, Fredholm alternative, nonlinear elliptic problems with Wentzell boundary conditions, submitted.

[26] C. Giorgi, M. Grasselli, V. Pata, Uniform attractors for a phase-field model with memory and quadratic nonlinearity, Indiana Univ. Math. J. 48 (1999), 1395-1445.

[27] C.G. Gal, A. Miranville, Uniform global attractors for non-isothermal Cahn-Hilliard equations with dynamic boundary conditions, Nonlinear Anal. Series B: Real World Applications, to appear.

[28] C.G. Gal, A. Miranville, Robust exponential attractors for non-isothermal Cahn-Hilliard equations with dynamic boundary conditions, submitted.

[29] S. Gatti, A. Miranville, Asymptotic behavior of phase-field system with dynamic boundary conditions, in "Differential Equations Inverse and Direct Problems"(A. Favini, A. Lorenzi, Eds.), Ser. Lect. Notes Pure Appl. Math. 251, 149-170, Chapman \& Hall/CRC, Boca Raton, 2006.

[30] M. Grasselli, H. Petzeltovà, G. Schimperna, Long time behavior of solutions to the Caginalp system with singular potential, Z. Anal. Anwendungen 25 (2006), 51-72.

[31] M. Grasselli, H. Petzeltovà, G. Schimperna, Asymptotic behaviour of a nonisothermal viscous Cahn-Hilliard equation with inertial term, Journal of Differential Equations 239 (2007), 38-60.

[32] G. Ruiz Goldstein, Derivation and interpretation of general boundary conditions, Adv. Differential Equations 11 (2006), 457-480.

[33] M. Gurtin, Generalized Ginzburg-Landau and Cahn-Hilliard equations based on a microforce balance, Phys. D92 (1996), 178-192.

[34] A. Jiménez-Casas, A. Rodríguez-Bernal, Asymptotic behaviour for a phase field model in higher order Sobolev spaces, Rev. Mat. Complut. 15 (2002), 213-248.

[35] V. K. Kalantarov, On the minimal global attractor of a system of phase field equations (Russian), Zap. Nauchn. Sem. Leningrad. Otdel. Mat. Inst. Steklov. (LOMI) 188 (1991), Kraev. Zadachi Mat. Fiz. i Smezh. Voprosy Teor. Funktsii. 22, 70-86, 186 [translation in J. Math. Sci. 70 (1994), no. 3, 1767-1777].

[36] O. V. Kapustyan, An attractor of a semiflow generated by a system of phase-field equations without uniqueness of the solution (Ukrainian), Ukraïn. Mat. Zh. 51 (1999), 1006-1009 [Translation in Ukrainian Math. J. 51 (1999), no.7, 1135-1139 (2000)].

[37] R. Kenzler, F. Eurich, Ph. Maass, B. Rinn, J. Schropp, E. Bohl, W. Dieterich, Phase separation in confined geometries: Solving the Cahn-Hilliard equation with generic boundary conditions, Computer Phys. Comm. 133 (2001), 139-157.

[38] N. Kenmochi, M. Niezgodka and I. Pawlow, Subdifferential operator approach to the CahnHilliard equations with constraint, J. Diff. Equations 117 (1995), 320-356.

[39] Ph. Laurençot, Long-time behaviour for a model of phase-field type, Proc. Roy. Soc. Edinburgh Sect.A 126 (1996), 167-185.

[40] O. A. Ladyzenskaya, O. A. Solonnikov and N. N. Uraltseva, Linear and quasilinear equations of parabolic type, translated from russiona, AMS, Providence, RI, (1967). 
[41] A. Miranville, S. Zelik, Exponential attractors for the Cahn-Hilliard equation with dynamical boundary conditions, Math. Models Appl. Sci. 28 (2005), 709-735.

[42] A. Miranville, S. Zelik, Robust exponential attractors for singularly perturbed phase-field type equations, Electron. J. Differential Equations 63 (2002), 1-28.

[43] A. Novick-Cohen, The Cahn-Hilliard equation: Mathematical and modeling perspectives, Adv. Math. Sci. Appl. 8 (1998), 965-985.

[44] A. Novick-Cohen, On the viscous Cahn-Hilliard equation, in "Material instabilities in continuum mechanics (Edinburgh, 1985-1986)", Oxford Sci. Publ., 329-342, Oxford Univ. Press, New York, 1988.

[45] J. Prüss, R. Racke, S. Zheng: Maximal regularity and asymptotic behavior of solutions for the Cahn Hilliard equation with dynamic boundary conditions, Ann. Mat. Pura Appl. (4) 185 (2006), 627-648.

[46] J. Prüss, M. Wilke, Maximal $\mathrm{L}^{p}$ regularity for the Cahn Hilliard equation with non-constant temperature and dynamic boundary conditions, in "Partial differential equations and functional analysis", Oper. Theory Adv. Appl. 168, 209-236, Birkhäuser, Basel, 2006.

[47] V. Pata, S. Zelik, A result on the existence of global attractors for semigroups of closed operators, Commun. Pure Appl. Anal. 6 (2007), 481-486.

[48] R. Racke, S. Zheng, The Cahn-Hilliard equation with dynamical boundary conditions, Adv. Differential Equations 8 (2003), 83-110.

[49] N. Sato, T. Aiki, Phase field equations with constraints under nonlinear dynamic boundary conditions, Commun. Appl. Anal. 5 (2001), 215-234.

[50] G. Schimperna, Abstract approach to evolution equations of phase field type and applications, J. Differential Equations 164 (2000), 395-430.

[51] R. Temam, Infinite-Dimensional Dynamical Systems in Mechanics and Physics, SpringerVerlag, New York, 1997.

[52] $\mathrm{H}$. Wu, S. Zheng, Convergence to equilibrium for the Cahn-Hilliard equation with dynamic boundary conditions, J. Differential Equations 204 (2004), 511-531.

[53] Z. Zhang, Asymptotic behavior of solutions to the phase-field equations with Neumann boundary conditions, Commun. Pure Appl. Anal. 4 (2005), 683-693.

[54] S. Zheng, Asymptotic behavior of solutions to the Cahn-Hilliard equations, Appl. Anal. 3 (1986), 165-184.

[55] S. Zheng, Nonlinear parabolic equations and hyperbolic-parabolic coupled systems, Pitman Monographs Surv. Pure Appl. Math. 76, Longman; John Wiley \& Sons, New York (1995).

[56] S. Zheng, Nonlinear evolution equations, Chapman \& Hall/CRC, Boca Raton, FL, 2004.

Department of Mathematics, University of Missouri, Columbia, MO65211, USA

E-mail address: ciprian@math.missouri.edu 